\title{
Stellar parameters for Pop II A-type stars from IUE spectra and new-ODF ATLAS9 model atmospheres ${ }^{\star}$
}

\author{
F. Castelli ${ }^{1}$ and C. Cacciari ${ }^{2}$ \\ 1 CNR and Osservatorio Astronomico di Trieste, via G. B. Tiepolo 11, 34131 Trieste, Italy \\ 2 Osservatorio Astronomico di Bologna, via Ranzani 1, 40127 Bologna, Italy \\ e-mail: cacciari@bo.astro.it
}

Received 17 July 2001 / Accepted 10 October 2001

\begin{abstract}
Stellar parameters for twenty-seven field horizontal branch A-type stars, a post-AGB star $(\mathrm{BD}+322188)$, and a possible cool sdB star (BD +00 0145) were obtained by fitting the whole IUE energy distributions taken from the IUE-INES archive to the ultraviolet energy distributions predicted by new-ODF ATLAS9 model atmospheres, which include the Lyman- $\alpha \mathrm{H}-\mathrm{H}^{+}$and $\mathrm{H}-\mathrm{H}$ quasi-molecular absorptions near $1400 \AA$ and $1600 \AA$. The sample of stars was extensively studied by Kinman et al. (2000), who derived stellar parameters for them by using visual observations and also an ultraviolet color index. The effective temperatures obtained by fitting the IUE spectra to the new-ODF models agree with $T_{\text {eff }}$ derived by Kinman et al. (2000) for most of the stars in the sample. The gravities from UV agree with those from Kinman et al. (2000) for stars hotter than about $8700 \mathrm{~K}$, while they are lower, on average, by 0.3 dex for the cooler stars. The same discrepancy is present when $\log g$ from the ultraviolet energy distribution is compared with $\log g$ from the visible energy distribution. The difference is insensitive to reddening, microturbulent velocity, metallicity, or mixing-length parameter for the treatment of the convection.
\end{abstract}

Key words. stars: atmospheres - stars: fundamental parameters - ultraviolet: stars

\section{Introduction}

One of the methods for deriving stellar atmospheric parameters $T_{\text {eff }}$ and $\log g$ is the comparison of the observed and computed energy distributions. However, when this method was applied to the ultraviolet flux of metal deficient A-type stars, like $\lambda$ Boo stars and horizontal branch stars, some disagreement occurred between the observed and model fluxes. In particular, Huenemoerder et al. (1984) pointed out that the Kurucz (1979a) computed fluxes for Pop II stars bluer than $1600 \AA$ were brighter than the observed ones. Cacciari et al. (1987) derived stellar parameters for 16 Pop II stars by fitting the observed energy distributions to the Kurucz (1979b) models. There are seven stars in their sample hotter than $7500 \mathrm{~K}$. For three of them (HD 74721, HD 85504, HD 117880) the fitting interval started at $1411 \AA$, and for the other four stars (HD 60778, HD 86986, HD 109995, and HD 161817) it started at $1688 \AA$. The lower wavelength limits of the fitting interval were fixed on the basis of the failure of the fit at shorter wavelengths. The failure was ascribed

\footnotetext{
Send offprint requests to: F. Castelli,

e-mail: castelli@ts.astro.it

* Figures A.1 to A.15 are only available in electronic form at http://www . edpsciences.org
}

to shortcomings related with the ultraviolet opacity computations.

Improved opacity distribution functions (ODFs), based on both atomic and molecular lines, were later computed by Kurucz (1990) (hereafter old-ODFs) and new grids of models were produced (Kurucz 1993; Castelli 1999). We show in this paper that the improved fluxes compared to those used by Huenemoerder et al. (1984) are no longer brighter than the observed ones for $\lambda<1600 \AA$, but that they are still not able to reproduce the shortwavelength IUE region where the wide absorptions at $1400 \AA$ and $1600 \AA$, observed in $\lambda$ Boo stars and in Pop II A-type stars (Jaschek et al. 1985; Holweger et al. 1994), are present. These absorptions were the cause for the failure of the fit performed by Cacciari et al. (1987) for $\lambda<1400 \AA$ in the hotter stars and for $\lambda<1600 \AA$ in the cooler stars. In fact, the depressions were not included in the models because their origin was unknown until Holweger et al. (1994) demonstrated that they are due to quasi-molecular absorptions of the atomic hydrogen in the ground state perturbed by collisions with protons and other neutral hydrogen atoms.

A successive set of ODFs (hereafter new-ODFs) including the Lyman- $\alpha \mathrm{H}-\mathrm{H}$ and $\mathrm{H}-\mathrm{H}^{+}$quasi-molecular absorptions was computed by Castelli \& Kurucz (2001). 
The collisionally perturbed profiles computed in a semiclassical way by Allard et al. (1998) were added in the codes. More details on the synthetic Lyman- $\alpha$ profile can be found in Castelli \& Kurucz (2001), who tested the newODF models on $\lambda$ Boo. They derived stellar parameters for $\lambda$ Boo from the IUE interval 1300-1900 $\AA$ which do not differ more than $100 \mathrm{~K}$ in $T_{\text {eff }}$ and 0.1 dex in $\log g$ from the parameters derived by fitting the visible energy distribution.

Because the intensity and shape of the $\mathrm{H}-\mathrm{H}^{+}$and $\mathrm{H}-\mathrm{H}$ quasi-molecular absorptions strongly depend on the stellar parameters, we extend in this paper the analysis performed by Castelli \& Kurucz (2001) for $\lambda$ Boo to the sample of metal-poor A-type stars studied by Kinman et al. (2000, hereafter KCC) in order to compare the parameters derived by them with those derived from the whole ultraviolet energy distribution. KCC analyzed the spectra of thirty-one low-metallicity, low-gravity A-type stars observed at Kitt Peak $(\lambda / \Delta \lambda \sim 15000)$ and at ESO $(\lambda / \Delta \lambda \sim 30000$ and 40000$)$. All the stars of the sample were classified by KCC as field horizontal branch, except BD +32 2188 (a post-AGB star) and BD +00 0145 (a possible cool sdB star). The stellar parameters were determined from Strömgren photometry, from the UV color index $(18-V)=-2.5\left(\log F_{1800}-\log F_{V}\right)$, from the visual energy distribution, when available, and also from the $\mathrm{H}_{\gamma}$ profile for the 22 stars observed at Kitt Peak. Furthermore, abundances for $\mathrm{Mg}$, Ti, and Fe were derived from the spectra. In addition, the reddening $E(B-V)$ was estimated for all the stars of the sample.

The sample of Pop II A-type stars analyzed in this paper is formed by twenty-nine out of the thirty-one stars discussed by KCC, because for two of them, BD + 252602 and HD 16456, no IUE observations are available. In order to derive $T_{\text {eff }}$ and $\log g$ from the ultraviolet spectra, we computed new-ODFs for the metallicities close to the $[\mathrm{Fe} / \mathrm{H}]$ values derived by $\mathrm{KCC}$ and we computed small grids of new-ODF models and new-ODF fluxes for microturbulent velocities close to those estimated for the stars by KCC. Then we fitted the IUE spectra extracted from the INES archive (INES 2000), after having dereddened them, both to the old-ODF and the new-ODF models in order to derive $T_{\text {eff }}$ and $\log g$. For a few stars we adjusted the reddening or/and metallicity and microturbulent velocity on the basis of the ultraviolet analysis. For each star, we show plots where the IUE fluxes are overimposed to the following three models: (1) the old-ODF model having parameters from KCC, (2) the old-ODF model and (3) the new-ODF model both having parameters derived by fitting the IUE spectra to the grid of models computed for the metallicity and the microturbulent velocity which approximate those of the given star.

\section{The ultraviolet spectra of the Pop II A-type stars}

Ultraviolet data are the low-resolution IUE spectra extracted from the version 3.0 of the INES (IUE newly extracted spectra) archive (INES 2000), as available at the website http://ines.vilspa.esa.es. We used only lowresolution, large-aperture spectra. When more than one image was available for a given wavelength range, we selected the best one according to the exposure classification code $(E C C)$. Highest weight was given to $E C C=500$. In order to obtain only one spectrum, the selected longwavelength spectrum was joined to the selected shortwavelength spectrum at $1978 \AA$. The stars and the adopted spectra, together with their $E C C$, are listed in Table 1. The $V$ magnitude given in Col. 2 of Table 1 was taken from KCC. The last four columns of Table 1 give the abundance $[\mathrm{Fe} / \mathrm{H}]$ relative to the Sun, the effective temperature $T_{\text {eff }}$, the gravity $\log g$, and the microturbulent velocity $\xi$, which were obtained by KCC from their analysis of the stars.

Not all the IUE spectra are of good quality and there are only four stars out of the twenty-nine of the sample which have spectra with $E C C=500$ for both shortand long-wavelength regions. However, for one of them, HD 106304, the flux of the long-wavelength region is much lower than that of the short-wavelength region (Fig. A.3, Appendix A), in spite of both spectra having been classified as "good" in accordance with their ECC. As a consequence, we have used only the short-wavelength energy distribution for our analysis of HD 106304. The example of HD 106304 may lead us to suspect that some difference between the flux levels of the short- and long-wavelength regions may exist also for other stars, although less conspicuous than that observed for HD 106304, all the more that most of the stars have spectra of different quality for the two ranges.

\section{The models}

\subsection{The old-ODF models}

The old-ODF models and the old-ODF fluxes used in this paper are those computed by Castelli (1999) and adopted by KCC. They were computed with the ATLAS9 code by using ODFs from Kurucz (1990). The solar abundances adopted for the old-ODFs, the old-ODF models and the old-ODF fluxes are those from Anders \& Grevesse (1989), except for iron when the $\alpha$-enhanced models are considered. For $\alpha$-enhanced ODFs, models, and fluxes, the iron solar abundance was assumed equal to $\log \left(N_{\mathrm{Fe}} / N_{\text {tot }}\right)=$ -4.53, according to Holweger et al. (1995).

\subsection{The new-ODF models}

New ODFs were computed for metallicities covering the values of the sample, i.e. $[\mathrm{M} / \mathrm{H}]=-1.0,-1.0 \mathrm{a},-1.5$, $-1.5 \mathrm{a},-2.0,-2.0 \mathrm{a},-2.5,-2.5 \mathrm{a}$, and $-3.0 \mathrm{a}$. Furthermore, new-ODFs for $[\mathrm{M} / \mathrm{H}]=-1.25 \mathrm{a},-1.75 \mathrm{a}$, and $-2.25 \mathrm{a}$ were obtained by interpolation. The symbol "a" near the metallicity indicates an enhancement $\left[\alpha / \alpha_{\odot}\right]=+0.4$ dex for the $\alpha$-elements $\mathrm{O}, \mathrm{Ne}, \mathrm{Mg}, \mathrm{Si}, \mathrm{S}, \mathrm{Ar}, \mathrm{Ca}$, and Ti. For each metallicity, ODFs were computed for microturbulent 
Table 1. The sample of the A-type stars, the adopted IUE images, and stellar parameters from KCC.

\begin{tabular}{|c|c|c|c|c|c|c|c|c|c|}
\hline Star & $V$ & image & $E C C$ & image & $E C C$ & {$[\mathrm{Fe} / \mathrm{H}]$} & $T_{\text {eff }}$ & $\log g$ & $\xi$ \\
\hline HD 2857 & 9.990 & SWP56216 & 500 & LWR08070 & 403 & {$[-1.73]$} & 7550 & 3.00 & 3.0 \\
\hline HD 4850 & 9.619 & SWP55936 & 500 & LWP31488 & 500 & {$[-1.27]$} & 8450 & 3.20 & 2.0 \\
\hline HD 8376 & 9.655 & SWP56274 & 500 & & & {$[-2.95]$} & 8150 & 3.30 & 1.0 \\
\hline HD 13780 & 9.811 & SWP56045 & 500 & LWP31406 & 501 & {$[-1.45]$} & 7950 & 3.10 & 2.0 \\
\hline HD 14829 & 10.306 & SWP23439 & 503 & LWP31398 & 503 & {$[-2.39]$} & 8900 & 3.20 & 2.0 \\
\hline HD 31943 & 8.262 & SWP55928 & 500 & LWP23521 & 402 & {$[-0.99]$} & 7900 & 3.20 & 4.0 \\
\hline HD 60778 & 9.103 & SWP07351 & 502 & LWR06342 & XX1 & {$[-1.49]$} & 8050 & 3.10 & 3.0 \\
\hline HD 74721 & 8.713 & SWP08061 & $\mathrm{n} / \mathrm{a}$ & LWR07028 & 501 & {$[-1.42]$} & 8900 & 3.30 & 4.0 \\
\hline HD 78913 & 9.285 & SWP45164 & 400 & LWP23522 & 502 & {$[-1.43]$} & 8500 & 3.25 & 2.0 \\
\hline HD 86986 & 8.000 & SWP03931 & $\mathrm{n} / \mathrm{a}$ & LWR03502 & $\mathrm{n} / \mathrm{a}$ & {$[-1.81]$} & 7950 & 3.20 & 2.5 \\
\hline HD 87047 & 9.725 & SWP54441 & 400 & LWP30460 & 500 & {$[-2.47]$} & 7850 & 3.10 & 2.0 \\
\hline HD 87112 & 9.717 & SWP54452 & 500 & & & {$[-1.46]$} & 9750 & 3.50 & 2.0 \\
\hline HD 93329 & 8.790 & SWP54451 & 500 & LWP30469 & 500 & {$[-1.32]$} & 8250 & 3.10 & 2.0 \\
\hline HD 106304 & 9.069 & SWP54443 & 500 & (LWP30462) & 500 & {$[-1.34]$} & 9750 & 3.50 & 2.0 \\
\hline HD 109995 & 7.630 & SWP03932 & $\mathrm{n} / \mathrm{a}$ & LWP03637 & 504 & {$[-1.72]$} & 8500 & 3.10 & 3.0 \\
\hline HD 117880 & 9.064 & SWP23442 & $\mathrm{X} 01$ & LWP03751 & X02 & {$[-1.64]$} & 9300 & 3.30 & 2.0 \\
\hline HD 128801 & 8.738 & SWP45161 & 500 & LWP22291 & 502 & {$[-1.45]$} & 10300 & 3.55 & 2.0 \\
\hline HD 130095 & 8.128 & SWP03921 & $\mathrm{n} / \mathrm{a}$ & LWR03505 & $\mathrm{n} / \mathrm{a}$ & {$[-1.87]$} & 9000 & 3.30 & 2.0 \\
\hline HD 130201 & 10.110 & SWP54449 & 500 & LWP30466 & 500 & {$[-1.00]$} & 8650 & 3.50 & 2.0 \\
\hline HD 139961 & 8.860 & SWP45165 & 400 & LWP23523 & 502 & {$[-1.71]$} & 8500 & 3.20 & 3.0 \\
\hline HD 161817 & 6.976 & SWP03923 & $\mathrm{n} / \mathrm{a}$ & LWR03506 & $\mathrm{n} / \mathrm{a}$ & {$[-1.55]$} & 7550 & 3.00 & 3.0 \\
\hline HD 167105 & 8.966 & SWP55803 & 500 & LWP24147 & 501 & {$[-1.56]$} & 9050 & 3.30 & 3.0 \\
\hline HD 180903 & 9.568 & SWP46057 & 402 & LWP30467 & 400 & {$[-1.45]$} & 7700 & 3.10 & 3.0 \\
\hline HD 202759 & $9.09 \mathrm{v}$ & SWP46056 & 300 & LWP24146 & 502 & {$[-2.12]$} & 7500 & 3.05 & 2.0 \\
\hline HD 213468 & 10.926 & SWP10663 & 302 & LWR09371 & $\mathrm{n} / \mathrm{a}$ & {$[-1.67]$} & 9150 & 3.30 & 2.0 \\
\hline HD 252940 & 9.098 & SWP56153 & 500 & LWP24148 & 502 & {$[-1.77]$} & 7550 & 2.95 & 3.5 \\
\hline $\mathrm{BD}+00145$ & 10.580 & SWP11203 & 502 & LWR09819 & $\mathrm{n} / \mathrm{a}$ & {$[-2.45]$} & 9700 & 4.00 & 2.0 \\
\hline $\mathrm{BD}+322188$ & 10.756 & SWP07852 & 502 & LWP30459 & 500 & {$[-1.11]$} & 10450 & 2.10 & 1.0 \\
\hline $\mathrm{BD}+422309$ & 10.771 & SWP23441 & 407 & & & {$[-1.63]$} & 8800 & 3.20 & 2.0 \\
\hline
\end{tabular}

velocities $\xi=0,1,2,4$, and $8 \mathrm{~km} \mathrm{~s}^{-1}$, in analogy with the old-ODFs computed by Kurucz (1990).

In the new-ODFs the Lyman- $\alpha \mathrm{H}-\mathrm{H}$ and $\mathrm{H}-\mathrm{H}^{+}$quasimolecular absorptions near $1600 \AA$ and $1400 \AA$ are considered and they are computed according to Allard et al. (1998). The solar abundances adopted for the new-ODFs, the new-ODF models and new-ODF fluxes are those from Grevesse et al. (1996). Modifications in the treatment of the overlapping lines at the end of the term series have slightly changed the shape of the flux computed just shortward of the Balmer discontinuity. More details about the new-ODFs can be found in Castelli \& Kurucz (2001).

Small grids of ATLAS9 models and fluxes were generated by using the new-ODFs in order to derive the stellar parameters from the fit of the IUE observations to the grids of synthetic fluxes. Also the final fitting model was directly computed with the ATLAS9 code. All the adopted models were computed with the option for the convection switched on, but with the option for the approximate overshooting switched off. The convection is treated with the mixing-length theory. The mixing-length to the pressure scale height ratio $L / H_{\mathrm{p}}$ was assumed to be 1.25 . The computed convective flux decreases with increasing $T_{\text {eff }}$, so that it becomes either negligible or equal to zero in the hottest models considered in this paper.
Figures 1 and 2 compare fluxes $F_{\lambda}$ computed from old-ODF models and new-ODF models. Figure 1a shows fluxes computed for $\log g=3.0,[\mathrm{M} / \mathrm{H}]=-1.50 \mathrm{a}$, and different $T_{\text {eff }}$ equal to $9000 \mathrm{~K}, 8500 \mathrm{~K}$, and $8000 \mathrm{~K}$. Figure $1 \mathrm{~b}$ shows fluxes computed for $T_{\text {eff }}=8000 \mathrm{~K}$, $[\mathrm{M} / \mathrm{H}]=-1.5 \mathrm{a}$, and different $\log g$ equal to $4.00 \mathrm{dex}$, 3.00 dex, and 2.00 dex. Figure 2 shows fluxes computed for $T_{\text {eff }}=8000 \mathrm{~K}, \log g=3.0$, and different metallicities $[\mathrm{M} / \mathrm{H}]$ equal to $-1.00 \mathrm{a},-1.50 \mathrm{a}$, and $-2.00 \mathrm{a}$. All the models displayed in Figs. 1 and 2 are computed with ODFs corresponding to a microturbulent velocity $\xi=2 \mathrm{~km} \mathrm{~s}^{-1}$. The differences between the old-ODF fluxes and newODF fluxes shortward $1600 \AA$ increase with decreasing $T_{\text {eff }}$, increasing gravity, and decreasing metallicity. This behaviour is well manifest in the IUE spectra showed in Figs. A.1-A.15 of Appendix A, where the stars are ordered by decreasing $T_{\text {eff }}$.

\section{The dependence of the ultraviolet spectral energy distribution on $T_{\text {eff }}, \log g$ and metallicity}

As was shown by Holweger et al. (1994) and by Allard et al. (1998), the ultraviolet flux of the metal-poor A-type stars in the region $1250-1900 \AA$ is strongly dependent on the model parameters. Castelli \& Kurucz (2001) showed 


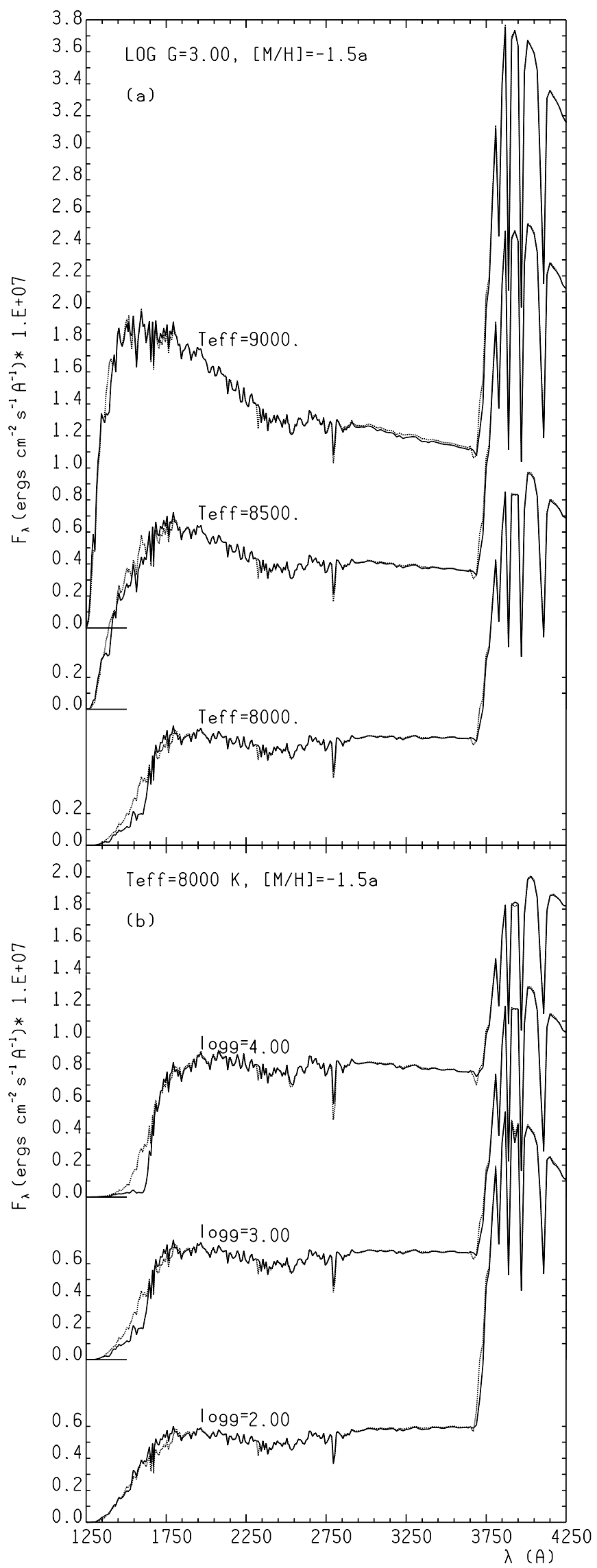

Fig. 1. Comparison of fluxes $F_{\lambda}$ computed from new-ODF models (full thick line) and old-ODF models (dashed line): a) for different $T_{\text {eff }}$; b) for different $\log g$.

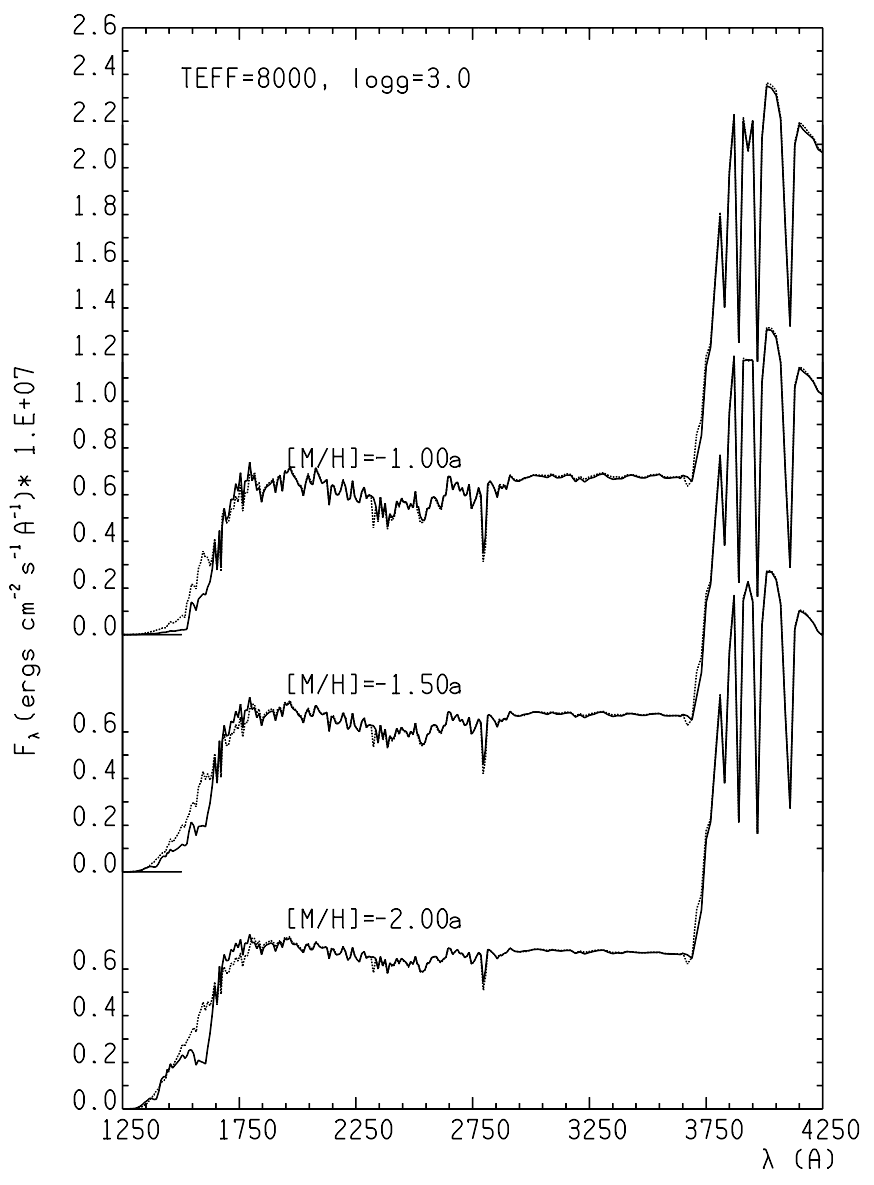

Fig. 2. Comparison of fluxes $F_{\lambda}$ computed from new-ODF models (full thick line) and old-ODF models (dashed line) for different metallicities $[\mathrm{M} / \mathrm{H}]$.

the dependence of the computed fluxes on the parameters for no $\alpha$-enhanced models and for models computed for the particular abundances of $\lambda$ Boo. In this paper we show the dependence of the computed ultraviolet fluxes on the parameters for the $\alpha$-enhanced models. Figures 3a and $3 \mathrm{~b}$ illustrate, for the range $1250-3000 \AA$, the variation of the synthetic fluxes as a function of effective temperature and gravity, respectively, when ODFs computed for $[\mathrm{M} / \mathrm{H}]=-1.50 \mathrm{a}$ are used. The fluxes computed for metallicities ranging from $-2.50 \mathrm{a}$ to $-1.00 \mathrm{a}$ at steps of $0.5 \mathrm{dex}$, and $T_{\text {eff }}=8500 \mathrm{~K}, \log g=3.0$ are shown in Fig. 3c for the same wavelength range. In each panel of Fig. 3 the fluxes are normalized to $5556 \AA$ in order to be consistent with the usual comparison between observed and computed fluxes. All the models displayed in Fig. 3 are computed with ODFs corresponding to a microturbulent velocity $\xi=2 \mathrm{~km} \mathrm{~s}^{-1}$.

Figure 3 points out the different behaviour of the energy distribution in the IUE short- and long-wavelength regions. In the range 1250-2000 $\AA$ the energy distribution depends on $T_{\text {eff }}$, on $\log g$, and also on the metallicity owing to the C I and Si I discontinuities at $1444 \AA$ and $1525 \AA$ respectively. On the contrary, the energy distribution in the 2000-3000 $\AA$ region weakly depends on the metallicity and it is hardly useful to fix both $T_{\text {eff }}$ and $\log g$ at the 
same time, owing to the similar dependence of the energy distribution on $T_{\text {eff }}$ and on $\log g$.

The comparison of Fig. 3 with Fig. 7 in Castelli \& Kurucz $(2001)^{1}$ indicates that the general behaviour is the same for the two sets of models, which were computed with different metallicities. The differences are mostly related with the different carbon and silicon abundances adopted in the model computations. For instance, when Fig. 3 is compared with Fig. 7 of Castelli \& Kurucz (2001), it shows that the 0.4 dex larger silicon abundance and the -1.1 dex lower carbon abundance, adopted in this paper, increase the Si I discontinuity at $1525 \AA$ and decrease both the C I discontinuity at $1444 \AA$ and the intensity of all the C I lines, in particular that of the blend observed at $1657 \AA$.

\section{The fitting procedure}

The fitting procedure is based on that described by Lane \& Lester (1984) in which the observed energy distribution is fitted to the model which yields the minimum rms difference. The search for the minimum rms difference is made by interpolating in the grid of computed fluxes. The computed fluxes are sampled in steps of $50 \mathrm{~K}$ in $T_{\text {eff }}$ and in steps of 0.1 dex in $\log g$, so the finer sampling was obtained by linear interpolation. The error $\sigma(\lambda)$ of the flux associated to the INES spectra was used at each wavelength to weight the square differences between the observed flux and the computed flux. In this way, the parameters derived by fitting a selected image to the models are almost independent from the limits of the wavelength interval adopted for the fit. Bad pixels were excluded from the fit.

For the fitting procedure, the IUE spectra were dereddened for the $E(B-V)$ listed in Col. 4 of Table 2. The reddening $E(B-V)$ was taken from KCC for all the stars, except HD 8376, HD 13780, HD 109995. The new values were fixed on the basis of the better agreement between the observed and computed ultraviolet fluxes yielded by them. The interstellar extinction, as a function of wavelength, was taken from Mathis (1990). We adopted $R_{V}=3.1$. The dereddened IUE fluxes and the computed fluxes were normalized at $5556 \AA$. The observed flux at $5556 \AA$ was obtained by means of the relation $\log F_{\lambda}=-0.400 \mathrm{~V}-8.456$ from Gray (1976, p. 202) and it was then dereddened according to the procedure used for the ultraviolet fluxes.

For each star, a grid of new-ODF models computed for a given metallicity $[\mathrm{M} / \mathrm{H}]$ and a given microturbulent velocity $\xi$ was selected for the fit. The metallicity $[\mathrm{M} / \mathrm{H}]$ is that listed in Col. 2 of Table 2. For all the stars, except HD 8376, HD 93329, and HD 117880, it approximates within 0.125 dex the iron abundance $[\mathrm{Fe} / \mathrm{H}]$ obtained by KCC and given in Col. 7 of Table 1. Because the abundance analysis performed by KCC indicated that all the

\footnotetext{
${ }^{1}$ The flux units in Fig. 7 in Castelli \& Kurucz (2001) are actually ergs cm $\mathrm{cm}^{-2} \mathrm{~s}^{-1} \AA^{-1} 10^{6}$ and not ergs $\mathrm{cm}^{-2} \mathrm{~s}^{-1} \AA^{-1} 10^{8}$; the same correction which replaces $10^{8}$ by $10^{6}$ has to be done in Figs. 1, 2, 4, 8, 11 and A.1. in the same paper.
}

stars, except $\mathrm{BD}+32$ 2188, have the $\mathrm{Mg}$ and $\mathrm{Ti}$ abundances enhanced, on average, by 0.4 dex over the iron, we started by assuming that all the alpha elements are equally enhanced. Therefore we adopted $\alpha$-enhanced ATLAS9 models and fluxes for all the stars, except BD +32 2188 .

The microturbulent velocity $\xi$ given in Col. 3 of Table 2 is based on that derived by KCC. Because ODFs are computed only for $\xi=0.0,1.0,2.0,4.0$ and $8.0 \mathrm{~km} \mathrm{~s}^{-1}$, we approximated the microturbulent velocities $\xi=2.5 \mathrm{~km} \mathrm{~s}^{-1}$, $3.0 \mathrm{~km} \mathrm{~s}^{-1}$, and $3.5 \mathrm{~km} \mathrm{~s}^{-1}$ obtained by KCC for some stars with $\xi=2.0 \mathrm{~km} \mathrm{~s}^{-1}$ or $\xi=4.0 \mathrm{~km} \mathrm{~s}^{-1}$, rather than interpolating the ODFs for the microturbulent velocity. In fact, the uncertainty in $\xi$ is not less than $1 \mathrm{~km} \mathrm{~s}^{-1}$. As explained in Sect. 8 in Kinman et al. (2000), the microturbulent velocity in KCC was derived from Fe I, Fe II, and $\mathrm{Ti}$ II lines by assuming that, for a given element, the abundance is independent of the equivalent widths. The uncertainty, however, both in the equivalent widths of the weak lines and in the log $g f$ values (especially for the lines of Ti II, which are the most numerous) severely limits this method of obtaining $\xi$. The adopted lines, their measured equivalent widths, the adopted $\log g f$ and their sources are given for each star in Tables 4 and 5 in Kinman et al. (2000). The value of $\xi$ derived from the equivalent widths was then refined by KCC by comparing the observed spectra against a series of synthetic spectra in which $\xi$ was sampled in steps of $1 \mathrm{~km} \mathrm{~s}^{-1}$; only in a few cases an intermediate step of $0.5 \mathrm{~km} \mathrm{~s}^{-1}$ was used.

Furthermore, for $\mathrm{BD}+00$ 145, HD 14829 and all the stars observed only at ESO (HD 4850, HD 13780, HD 78913, HD 106304, HD 130201, HD 213468) $\xi$ was assumed "a priori" to be equal to $2 \mathrm{~km} \mathrm{~s}^{-1}$, because there were too few lines in the spectra of these stars even for an estimate of $\xi$.

The comparison of the observed and computed ultraviolet energy distributions has led us to modify the starting parameters for some stars. In particular the changes are: HD 8376: $[\mathrm{M} / \mathrm{H}]=-2.50$, instead of $[\mathrm{M} / \mathrm{H}]=-2.95 \mathrm{a}$; HD 13780: $[\mathrm{M} / \mathrm{H}]=-1.50$, instead of $[\mathrm{M} / \mathrm{H}]=-1.50 \mathrm{a}$; HD 93329: $[\mathrm{M} / \mathrm{H}]=-1.50 \mathrm{a}$, instead of $[\mathrm{M} / \mathrm{H}]=-1.25 \mathrm{a}$; HD 117880: $[\mathrm{M} / \mathrm{H}]=-1.50 \mathrm{a}, \xi=2 \mathrm{~km} \mathrm{~s}^{-1}$, instead of $[\mathrm{M} / \mathrm{H}]=-1.75 \mathrm{a}$, and $\xi=4 \mathrm{~km} \mathrm{~s}^{-1}$.

The metallicity was modified mostly on the basis of the comparison between the observed and computed energy distributions shortward of $1500 \AA$, where the intensity of the emitted flux is related with the size of the Si I discontinuity at $1525 \AA$. Also for HD 87047, a silicon abundance higher than the adopted one of -6.59 dex, could have improved the agreement between observations and computations for $\lambda<1500 \AA$ (Fig. A.12, Appendix A). However, for all the stars, a detailed abundance analysis is needed in order to fix the silicon abundance best reproducing the observations.

In Table 2 we list the parameters derived by fitting the short-wavelength part (1200-1978 $\AA$ ) of the UV energy distribution (Cols. 5 and 6 ), the long-wavelength part (1979-3300 A) (Cols. 7 and 8), and the whole IUE spectrum (1200-3300 $\AA$ ) (Cols. 9 and 10) to the new-ODF 


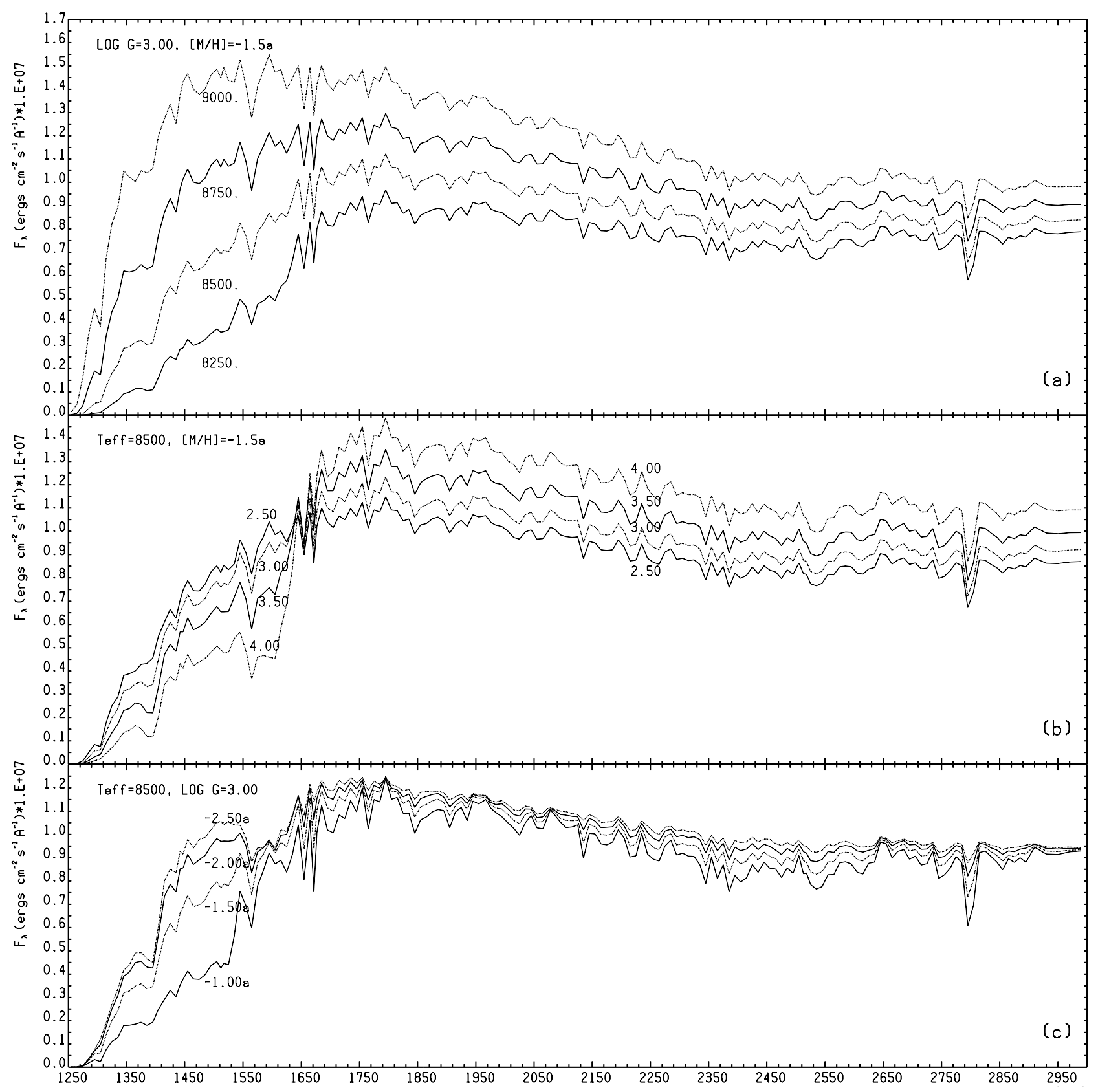

Fig. 3. The dependence of the ultraviolet flux on the model parameters in the region 1250-3000 $\AA$. a) $\log g=3.0,[\mathrm{M} / \mathrm{H}]=-1.5 \mathrm{a}$ and $T_{\text {eff }}=9000 \mathrm{~K}, 8750 \mathrm{~K}, 8500 \mathrm{~K}$, and $8250 \mathrm{~K}$. b) $T_{\text {eff }}=8500 \mathrm{~K},[\mathrm{M} / \mathrm{H}]=-1.5 \mathrm{a}$, and $\log g=2.50,3.00,3.50$, and 4.0 . c) $T_{\text {eff }}=8500 \mathrm{~K}, \log g=3.00$, and $[\mathrm{M} / \mathrm{H}]=-2.5 \mathrm{a},-2.0 \mathrm{a},-1.5 \mathrm{a}$ and $-1.0 \mathrm{a}$. The ordinate is the absolute flux $F_{\lambda}$ at the star surface. The fluxes are normalized at $5556 \AA$.

models. The parameters derived from the entire IUE energy distribution are very similar to those derived from the short-wavelength range, confirming the results of Fig. 3 that shows the stronger dependence of the parameters on the short-wavelength spectrum.

The differences in $T_{\text {eff }}$ and $\log g$ obtained from the short- and long-wavelength regions are, on average, on the order of $150 \mathrm{~K}$ in $T_{\text {eff }}$ and 0.3 dex in $\log g . T_{\text {eff }}$ from the 1200-1978 $\AA$ spectrum is generally higher than that from the 1979-3300 $\AA$ spectrum. The differences in the parameters are due to the difficulty in obtaining both $T_{\text {eff }}$ and $\log g$ from the IUE long-wavelength region and also probably to possible inconsistencies between the shortand long IUE spectra (Sect. 2) or to some inaccuracy in the models. For instance, the models were computed with "a priori" abundances for all the elements, except magnesium, titanium, and iron for which the actual abundances derived by KCC were used; in addition, the models are affected by a lower line blanketing than the real energy distributions, owing to several missing lines, in particular in the UV.

For each star, a grid of old-ODF fluxes computed for the same metallicity and microturbulent velocity adopted for the new-ODF fluxes (Table 2, Cols. 2 and 3) was used 
Table 2. The parameters derived from the fit of the IUE fluxes to the new-ODF models.

\begin{tabular}{|c|c|c|c|c|c|c|c|c|c|}
\hline \multirow{2}{*}{$\begin{array}{l}\text { Star } \\
\text { (1) }\end{array}$} & \multirow{2}{*}{$\begin{array}{r}{[M / H]} \\
(2)\end{array}$} & \multirow{2}{*}{$\begin{array}{l}\xi \\
(3)\end{array}$} & \multirow{2}{*}{$\begin{array}{l}E(B-V) \\
(4)\end{array}$} & \multirow{2}{*}{\multicolumn{2}{|c|}{$\begin{array}{l}T_{\text {eff }} \quad \log g \\
1200-1978 \AA \\
(5)\end{array}$}} & \multirow{2}{*}{\multicolumn{2}{|c|}{$\begin{array}{c}T_{\text {eff }} \quad \log g \\
1979-3300 \AA \\
(7) \quad(8)\end{array}$}} & \multicolumn{2}{|c|}{$\begin{array}{c}T_{\text {eff }} \quad \log g \\
1200-3300 \AA\end{array}$} \\
\hline & & & & & & & & (9) & $(10)$ \\
\hline HD 2857 & {$[-1.75 \mathrm{a}]$} & $4.0(3.0)$ & 0.022 & 7650 & 2.5 & 7550 & 3.0 & 7600 & 2.8 \\
\hline HD 4850 & {$[-1.25 \mathrm{a}]$} & 2.0 & 0.009 & 8450 & 2.8 & 8500 & 2.2 & 8450 & 2.7 \\
\hline HD 8376 & {$\left[-2.50^{1}\right]$} & 1.0 & $0.020^{2}$ & 8050 & 2.6 & & & & \\
\hline HD 13780 & {$[-1.50]$} & 2.0 & $0.000^{2}$ & 7900 & 2.7 & 7650 & 2.9 & 7900 & 2.7 \\
\hline HD 14829 & {$[-2.50 \mathrm{a}]$} & 2.0 & 0.018 & 8900 & 3.1 & 8900 & 3.1 & 8900 & 3.1 \\
\hline HD 31943 & {$[-1.00 \mathrm{a}]$} & 4.0 & 0.006 & 7850 & 3.1 & 7900 & 3.0 & 7850 & 3.1 \\
\hline HD 60778 & {$[-1.50 \mathrm{a}]$} & $4.0(3.0)$ & 0.028 & 8250 & 2.9 & 8400 & 2.6 & 8250 & 2.9 \\
\hline HD 74721 & {$[-1.50 \mathrm{a}]$} & $2.0(4.0)$ & 0.012 & 8800 & 3.2 & 8550 & 3.8 & 8800 & 3.2 \\
\hline HD 78913 & {$[-1.50 \mathrm{a}]$} & 2.0 & 0.034 & 8750 & 2.9 & 8600 & 2.9 & 8700 & 2.8 \\
\hline HD 86986 & {$[-1.75 \mathrm{a}]$} & $2.0(2.5)$ & 0.022 & 8100 & 2.7 & 7650 & 3.3 & 8100 & 2.8 \\
\hline HD 87047 & {$[-2.50 \mathrm{a}]$} & 2.0 & 0.006 & 7900 & 2.7 & 7800 & 2.9 & 7900 & 2.8 \\
\hline HD 87112 & {$[-1.50 \mathrm{a}]$} & 2.0 & 0.003 & 9700 & 3.6 & & & & \\
\hline HD 93329 & {$[-1.50 \mathrm{a}]$} & 2.0 & 0.014 & 8250 & 2.8 & 8250 & 2.9 & 8250 & 2.9 \\
\hline HD 106304 & {$[-1.25 \mathrm{a}]$} & 2.0 & 0.038 & 9600 & 3.5 & & & & \\
\hline HD 109995 & {$[-1.75 \mathrm{a}]$} & $2.0(3.0)$ & $0.010^{2}$ & 8500 & 3.1 & 8250 & 3.3 & 8500 & 3.0 \\
\hline HD 117880 & {$[-1.50 \mathrm{a}]$} & 2.0 & 0.077 & 9350 & 3.5 & 9000 & 4.0 & 9350 & 3.3 \\
\hline HD 128801 & {$[-1.50 \mathrm{a}]$} & 2.0 & 0.010 & 10200 & 3.5 & 10000 & 3.7 & 10200 & 3.5 \\
\hline HD 130095 & {$[-1.75 \mathrm{a}]$} & 2.0 & 0.072 & 9100 & 3.1 & 9200 & 2.9 & 9100 & 3.2 \\
\hline HD 130201 & {$[-1.00 \mathrm{a}]$} & 2.0 & 0.035 & 8900 & 2.5 & 8750 & 2.8 & 8900 & 2.5 \\
\hline HD 139961 & {$[-1.75 \mathrm{a}]$} & $4.0(3.0)$ & 0.051 & 8600 & 3.0 & 8550 & 2.6 & 8600 & 2.8 \\
\hline HD 161817 & {$[-1.50 \mathrm{a}]$} & $4.0(3.0)$ & 0.000 & 7600 & 2.6 & 7250 & 3.1 & 7600 & 2.7 \\
\hline HD 167105 & {$[-1.50 \mathrm{a}]$} & $2.0(3.0)$ & 0.024 & 8900 & 3.2 & 8950 & 2.8 & 9000 & 3.1 \\
\hline HD 180903 & {$[-1.50 \mathrm{a}]$} & $4.0(3.0)$ & 0.098 & 7850 & 2.8 & 7550 & 3.0 & 7800 & 2.9 \\
\hline HD 202759 & {$[-2.00 \mathrm{a}]$} & 2.0 & 0.072 & 7500 & 2.9 & 7400 & 2.8 & 7500 & 2.8 \\
\hline HD 213468 & {$[-1.75 \mathrm{a}]$} & 2.0 & 0.008 & 9100 & 3.4 & 8900 & 3.6 & 9100 & 3.3 \\
\hline HD 252940 & {$[-1.75 \mathrm{a}]$} & $4.0(3.5)$ & 0.048 & 7650 & 2.6 & 7500 & 2.8 & 7650 & 2.7 \\
\hline $\mathrm{BD}+00145^{a}$ & {$[-2.50 \mathrm{a}]$} & 2.0 & 0.018 & 9900 & 3.2 & 9750 & 3.0 & 9900 & 3.1 \\
\hline $\mathrm{BD}+322188$ & {$[-1.00]$} & 1.0 & 0.007 & 10300 & 2.1 & 10250 & 2.1 & 10300 & 2.2 \\
\hline $\mathrm{BD}+422309$ & {$[-1.75 \mathrm{a}]$} & 2.0 & 0.013 & 8750 & 3.0 & & & & \\
\hline
\end{tabular}

${ }^{1}[\mathrm{Fe} / \mathrm{H}]$ from KCC is $[-2.95]$ for HD 8376.

$2 E(B-V)$ from KCC is 0.041 for HD 8376, 0.014 for HD 13780, and 0.022 for HD 109995 .

in order to derive parameters from the old-ODF models by means of the fitting procedure. Table 3 compares the parameters from the new-ODF models and the whole IUE wavelength region, the parameters from the old-ODF models and the whole IUE wavelength region, and the parameters from KCC. The stars are ordered by decreasing $T_{\text {eff }}$, as they were derived from the new-ODF models, and as they are ordered in Appendix A, where the observed energy distributions are compared with energy distributions computed from the old-ODF models, from the newODF models, and from KCC. This order allows a better estimate of the dependence of the ultraviolet energy distribution on $T_{\text {eff }}$, in particular of the $\mathrm{H}-\mathrm{H}^{+}$and $\mathrm{H}-\mathrm{H}$ quasi-molecular absorptions near $1400 \AA$ and $1600 \AA$.

Table 3 shows that, for most of the stars, the parameters from old-ODF models and new-ODF models are the same within the uncertainty of the fit which is on the order of $50 \mathrm{~K}$ in $T_{\text {eff }}$ and 0.1 dex in log $g$. Instead, Figs. A.1-A.15 in Appendix A show that the short-wavelength energy distributions are reproduced by the new-ODF models much better, especially for stars cooler than $8000 \mathrm{~K}$. Therefore, in spite of the parameters being almost the same, the rms from the fit is lower when the new-ODF models are used. In general, the gravities from the old-ODF models are higher than those from the new-ODF models. The largest difference, which amounts to 0.3 dex, occurs for $\mathrm{BD}+00$ 145, HD 117880, and HD 213468. Because no model is able to reproduce the core of Lyman- $\alpha$ of $\mathrm{BD}+00185$, the gravity is different depending on the wavelength interval (1200-3300 $\AA$ or $1300-3300 \AA$ ) used for the fit. In the second case, the model reproduces the observed absorption at $1400 \AA$ well, which is instead predicted too low in the first case. Therefore we adopted for $\mathrm{BD}+00185$ the parameters derived by fitting the 1300$3300 \AA$ A region.

The last two columns of Table 3 list the parameters found by KCC. Figure 4 illustrates, in the upper panel, the differences between $T_{\text {eff }}$ from $\mathrm{KCC}$ and $T_{\text {eff }}$ from the IUE fluxes and new-ODF models and, in the lower panel, the differences between $\log g$ from $\mathrm{KCC}$ and $\log g$ from 
Table 3. The comparison of parameters from new-ODF, old-ODF models and KCC.

\begin{tabular}{|c|c|c|c|c|c|c|c|c|c|}
\hline Star & $E(B-V)$ & {$[M / H]$} & $\xi$ & $\begin{array}{l}T_{\text {eff }} \\
\text { new- }\end{array}$ & $\begin{array}{l}\log g \\
\text { Fs }\end{array}$ & $\begin{array}{l}T_{\text {eff }} \\
\text { old- }\end{array}$ & $\begin{array}{l}\text { og } g \\
\text { s }\end{array}$ & $T_{\text {eff }}$ & $\log g$ \\
\hline $\mathrm{BD}+322188$ & 0.007 & {$[-1.00]$} & 1.0 & 10300 & 2.2 & 10300 & 2.2 & 10450 & 2.10 \\
\hline HD 128801 & 0.010 & {$[-1.50 \mathrm{a}]$} & 2.0 & 10200 & 3.5 & 10200 & 3.5 & 10300 & 3.55 \\
\hline $\begin{array}{l}\mathrm{BD}+00145 \\
\# 1\end{array}$ & 0.018 & {$[-2.50 \mathrm{a}]$} & 2.0 & $\begin{array}{l}9900 \\
9850\end{array}$ & $\begin{array}{l}3.2 \\
3.8\end{array}$ & $\begin{array}{l}9900 \\
9800\end{array}$ & $\begin{array}{l}2.9 \\
4.1\end{array}$ & 9700 & 4.00 \\
\hline HD 87112 & 0.003 & {$[-1.50 \mathrm{a}]$} & 2.0 & 9700 & 3.6 & 9650 & 3.8 & 9750 & 3.50 \\
\hline HD 106304 & 0.038 & {$[-1.25 \mathrm{a}]$} & 2.0 & 9600 & 3.5 & 9600 & 3.5 & 9750 & 3.50 \\
\hline HD 117880 & 0.077 & {$[-1.50 \mathrm{a}]$} & 2.0 & 9350 & 3.3 & 9300 & 3.6 & 9300 & 3.30 \\
\hline HD 213468 & 0.008 & {$[-1.75 \mathrm{a}]$} & 2.0 & 9100 & 3.3 & 9050 & 3.6 & 9150 & 3.30 \\
\hline HD 130095 & 0.072 & {$[-1.75 \mathrm{a}]$} & 2.0 & 9100 & 3.2 & 9100 & 3.3 & 9000 & 3.30 \\
\hline HD 167105 & 0.024 & {$[-1.50 \mathrm{a}]$} & 2.0 & 9000 & 3.1 & 9000 & 3.2 & 9050 & 3.30 \\
\hline HD 14829 & 0.018 & {$[-2.50 \mathrm{a}]$} & 2.0 & 8900 & 3.1 & 8900 & 3.1 & 8900 & 3.20 \\
\hline HD 130201 & 0.035 & {$[-1.00 \mathrm{a}]$} & 2.0 & 8900 & 2.5 & 8900 & 2.6 & 8650 & 3.50 \\
\hline HD 74721 & 0.012 & {$[-1.50 \mathrm{a}]$} & 2.0 & 8800 & 3.2 & 8800 & 3.3 & 8900 & 3.30 \\
\hline $\mathrm{BD}+422309$ & 0.013 & {$[-1.75 \mathrm{a}]$} & 2.0 & 8750 & 3.0 & 8700 & 3.2 & 8800 & 3.20 \\
\hline HD 78913 & 0.034 & {$[-1.50 \mathrm{a}]$} & 2.0 & 8700 & 2.8 & 8700 & 2.9 & 8500 & 3.25 \\
\hline HD 139961 & 0.051 & {$[-1.75 \mathrm{a}]$} & 4.0 & 8600 & 2.8 & 8600 & 2.8 & 8500 & 3.20 \\
\hline HD 109995 & 0.010 & {$[-1.75 \mathrm{a}]$} & 2.0 & 8500 & 3.0 & 8450 & 3.1 & 8500 & 3.10 \\
\hline HD 4850 & 0.009 & {$[-1.25 \mathrm{a}]$} & 2.0 & 8450 & 2.7 & 8400 & 2.9 & 8450 & 3.20 \\
\hline HD 93329 & 0.014 & {$[-1.50 \mathrm{a}]$} & 2.0 & 8250 & 2.9 & 8250 & 2.9 & 8250 & 3.10 \\
\hline HD 60778 & 0.028 & {$[-1.50 \mathrm{a}]$} & 4.0 & 8250 & 2.9 & 8200 & 3.1 & 8050 & 3.10 \\
\hline HD 86986 & 0.022 & {$[-1.75 \mathrm{a}]$} & 2.0 & 8100 & 2.8 & 8050 & 2.9 & 7950 & 3.20 \\
\hline HD 8376 & 0.020 & {$[-2.50]$} & 1.0 & 8050 & 2.6 & 8100 & 2.6 & 8150 & 3.30 \\
\hline HD 13780 & 0.000 & {$[-1.50]$} & 2.0 & 7900 & 2.7 & 7900 & 2.8 & 7950 & 3.10 \\
\hline HD 87047 & 0.006 & {$[-2.50 \mathrm{a}]$} & 2.0 & 7900 & 2.8 & 7850 & 2.9 & 7850 & 3.10 \\
\hline HD 31943 & 0.006 & {$[-1.00 \mathrm{a}]$} & 4.0 & 7850 & 3.1 & 7850 & 3.1 & 7900 & 3.20 \\
\hline HD 180903 & 0.098 & {$[-1.50 \mathrm{a}]$} & 4.0 & 7800 & 2.9 & 7800 & 2.9 & 7700 & 3.10 \\
\hline HD 252940 & 0.048 & {$[-1.75 \mathrm{a}]$} & 4.0 & 7650 & 2.7 & 7650 & 2.7 & 7550 & 2.95 \\
\hline HD 2857 & 0.022 & {$[-1.75 \mathrm{a}]$} & 4.0 & 7600 & 2.8 & 7600 & 2.8 & 7550 & 3.00 \\
\hline HD 161817 & 0.000 & {$[-1.50 \mathrm{a}]$} & 4.0 & 7600 & 2.7 & 7600 & 2.7 & 7550 & 3.00 \\
\hline HD 202759 & 0.072 & {$[-2.00 \mathrm{a}]$} & 2.0 & 7500 & 2.8 & 7550 & 2.8 & 7500 & 3.05 \\
\hline
\end{tabular}

${ }^{1}$ Parameters for a fitting range starting at $1300 \AA$ instead of at $1200 \AA$.

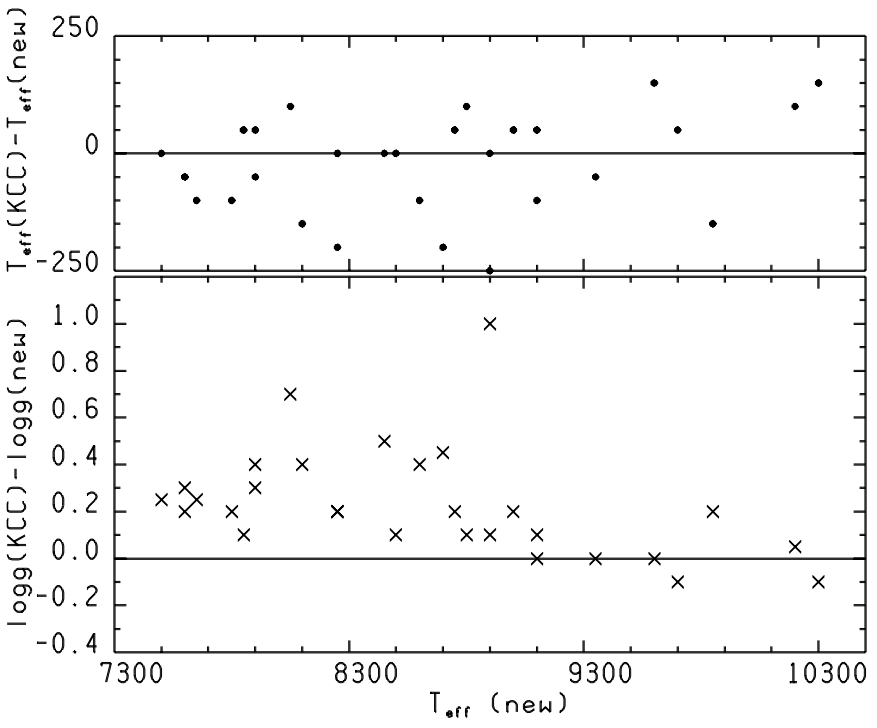

Fig. 4. Upper panel: $T_{\text {eff }}$ differences between KCC and newODF models as functions of $T_{\text {eff }}$, as derived from the new-ODF models. Lower panel: same as upper panel, for $\log g$. the IUE fluxes and new-ODF models. Both differences are plotted as a function of $T_{\text {eff }}$, as derived from the new-ODF models. The temperatures agree within $150 \mathrm{~K}$ for all the stars, except for HD $130201\left(\Delta T_{\text {eff }}=-250 \mathrm{~K}\right)$, HD 78913 $\left(\Delta T_{\text {eff }}=-200 \mathrm{~K}\right)$, and HD $60778\left(\Delta T_{\text {eff }}=-200 \mathrm{~K}\right)$. No trend of $\Delta T_{\text {eff }}$ with $T_{\text {eff }}$ is manifest. The gravities agree within 0.2 dex for the stars hotter than $8700 \mathrm{~K}$ (except HD 130201), but for the other stars the gravities from the whole IUE flux and new-ODF models are systamatically lower than those from KCC, with an average difference of about 0.3 dex.

\section{Parameters from the visible energy distribution for some stars of the sample}

The parameters adopted by KCC are the average of parameters obtained with different methods. They therefore represent a statistically "most likely" solution, but they mask the results obtained with a particular method. We wish to verify here how the parameters derived from the IUE spectra compare to those derived only from the visible 
energy distribution. Because there are 11 stars in our sample having visible spectrophotometry available in Philip \& Hayes (1983), we fitted these observations to the new-ODF models in order to derive both $T_{\text {eff }}$ and $\log g$ from the flux method for stars cooler than about $9000 \mathrm{~K}$ and only $T_{\text {eff }}$ for the hotter stars. In fact, as is illustrated in Fig. 5, the visible flux method is not well suited to derive the gravity for giant stars hotter than about $9000 \mathrm{~K}$. For these stars we will use the $\mathrm{H}_{\gamma}$ profile to derive $\log g$.

Figure 5 is a plot of the the Balmer discontinuity as a function of $T_{\text {eff }}$ for different $\log g$, where the Balmer jump is represented as a difference of two magnitudes. The first one is the magnitude averaged over five wavelengths in the UV ( $\lambda \lambda$ 3400, 3450, 3500, 3571, and $3636 \AA$ ) and the second one is the magnitude averaged over five wavelengths in the visible ( $\lambda \lambda 4036,4167,4255,4464$, and $4566 \AA)$. The wavelengths are the same of the observed energy distributions listed by Philip \& Hayes (1983) and used by them to estimate the errors of their scanner observations in the UV and in the blue, respectively. Figure 5 shows also the magnitude differences from Philip \& Hayes (1983) for the 11 stars listed in Table 4 . The error bars were obtained from the standard deviations quoted in their paper. They give an estimate of the error for the observed Balmer jump. The most uncertain data are those for BD +42 2309 and HD 14829.

Table 4 compares the parameters from the IUE spectra with those from the visible energy distribution and from $\mathrm{H}_{\gamma}$. The observed $\mathrm{H}_{\gamma}$ profiles are taken from KCC, while the synthetic profiles were computed with the BALMER9 code of Kurucz (1993). The observed $\mathrm{H}_{\gamma}$ profiles were fitted to a grid of profiles computed for different $\log g$, while $T_{\text {eff }}$ is that derived from the visible flux. Because $\mathrm{H}_{\gamma}$ for HD 14829 was not observed, we used the flux method to obtain also $\log g$ for this star. The differences in the parameters are plotted in Fig. 6. This figure is very similar to Fig. 4, where the parameters from UV and from KCC are compared. The large difference between $T_{\text {eff }}$ from the $\mathrm{UV}$ and from the visible for $\mathrm{BD}+422309$ is probably related with the poor observations for this star both in the $\mathrm{UV}$ and in the visible.

The conclusion is that gravities derived only from the visible flux distribution for stars cooler than about $8700 \mathrm{~K}$ are, on average, systematically larger by about 0.3 dex than those derived only from the ultraviolet flux distribution. For instance, Fig. 7 shows that there is no doubt about the need of gravities differing by $0.5 \mathrm{dex}$ in order to reproduce the short ultraviolet IUE spectrum and the visible energy distribution of HD 86986.

We remark that the parameters obtained by KCC only from the visible energy distribution (Table 7 in KCC) can not be compared with those listed in Cols. 7 and 8 of Table 4 , owing to the different $E(B-V)$ adopted in the two cases. In fact, $T_{\text {eff }}$ and $\log g$ in KCC correspond to the $E(B-V)$ yielding the best fit of the observed energy distribution to the models.

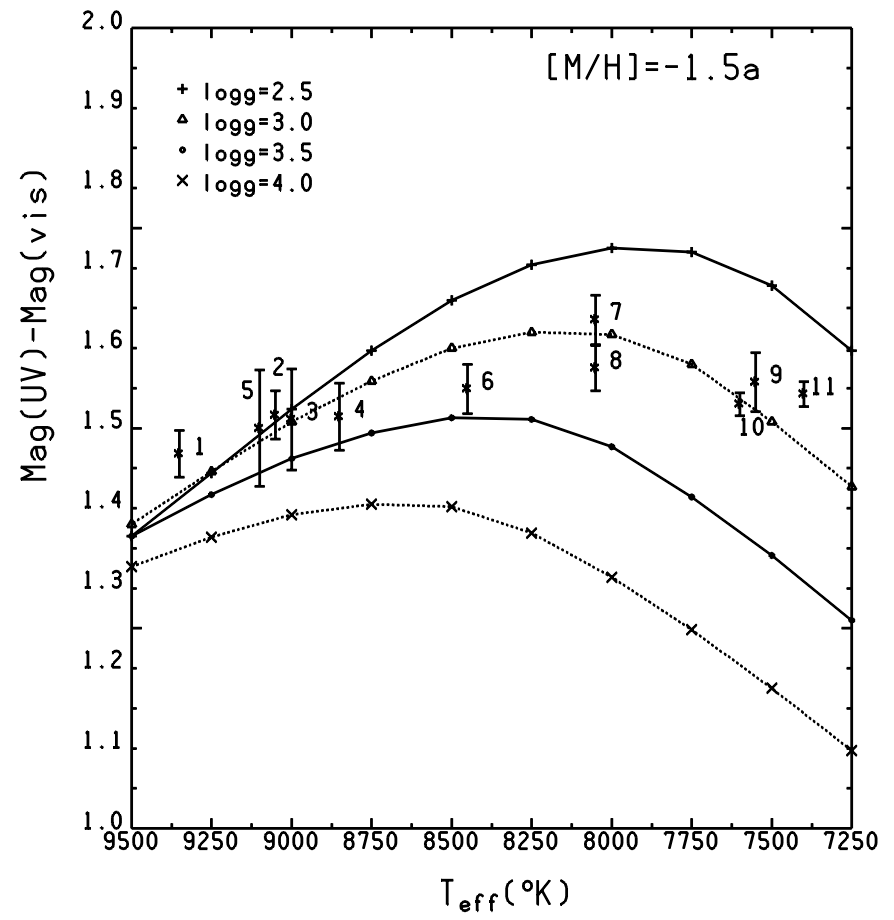

Fig. 5. The curves represent computed magnitude differences, Mag(UV)-Mag(vis), as function of $T_{\text {eff }}$ for different gravities and $[\mathrm{M} / \mathrm{H}]=-1.5 \mathrm{a}$. Mag $(\mathrm{UV})$ is the magnitude averaged over five wavelengths in the $3400-3636 \AA$ region and $\mathrm{Mag}$ (vis) is the magnitude averaged over five wavelengths in the 4036$4566 \AA$ region. The asterisks with error bars represent the magnitude differences for 11 stars obtained from the Philip \& Hayes (1983) scans. The correspondence of each number to each star is: $1=$ HD $117880,2=$ HD $130095,3=$ HD 14829 , $4=\mathrm{HD} 74721,5=\mathrm{BD}+42$ 2309, $6=\mathrm{HD} 109995,7=\mathrm{HD} 60778$, $8=$ HD 86986, $9=$ HD 2957, $10=$ HD 161817, $11=$ HD 202759.

\section{The effect of reddening, metallicity, microturbulent velocity and convection on the parameters}

\subsection{Reddening}

Table 5 lists reddening values taken from the literature for some stars of our sample. We converted the $E(b-y)$ from Hayes \& Philip (1988) to $E(B-V)$ by using the relation $E(B-V)=E(b-y) / 0.724$ from Crawford \& Mandwewala (1976). It is evident that $E(B-V)$ is a rather uncertain quantity for several stars. We therefore examined the effect of the reddening on the parameters of two stars, HD 2857 and HD 86986. By assuming different values of $E(B-V)$, we compared $T_{\text {eff }}$ and $\log g$ derived by fitting the dereddened IUE short-wavelength spectra to the models with $T_{\text {eff }}$ and $\log g$ derived by fitting the dereddened observed visible fluxes to the same grid of models. The results are given in Table 6. For HD 2857, $E(B-V)$ between 0.04 mag and $0.06 \mathrm{mag}$ gives $T_{\text {eff }}$ from the visible flux in agreement within $50 \mathrm{~K}$ with $T_{\text {eff }}$ from the ultraviolet flux, but there is always a difference on the order of $0.4-0.5$ dex between the corresponding gravities, whichever is $E(B-V)$. 
Table 4. Comparison of $T_{\text {eff }}$ and $\log g$ from ultraviolet and visible energy distributions and from $\mathrm{H}_{\gamma}$.

\begin{tabular}{lcccccccc}
\hline Star & $E(B-V)$ & {$[M / H]$} & $\xi$ & $\begin{array}{c}T_{\text {eff }} \\
\text { UV }\end{array}$ & & \multicolumn{1}{l}{$\log g$} & $\begin{array}{c}T_{\text {eff }} \\
\text { visible }\end{array}$ & $\begin{array}{r}\log g \\
\mathrm{H}_{\gamma}\end{array}$ \\
\hline HD 117880 & 0.077 & {$[-1.50 \mathrm{a}]$} & 2.0 & 9350 & 3.3 & 9400 & & 3.4 \\
HD 130095 & 0.072 & {$[-1.75 \mathrm{a}]$} & 2.0 & 9100 & 3.2 & 9050 & & 3.3 \\
HD 14829 & 0.018 & {$[-2.50 \mathrm{a}]$} & 2.0 & 8900 & 3.1 & 9000 & 3.1 & \\
HD 74721 & 0.012 & {$[-1.50 \mathrm{a}]$} & 2.0 & 8800 & 3.2 & 8850 & 3.3 & \\
BD +42 2309 & 0.013 & {$[-1.75 \mathrm{a}]$} & 2.0 & 8750 & 3.0 & 9100 & & 3.4 \\
HD 109995 & 0.010 & {$[-1.75 \mathrm{a}]$} & 2.0 & 8500 & 3.0 & 8450 & 3.4 & \\
HD 60778 & 0.028 & {$[-1.50 \mathrm{a}]$} & 4.0 & 8250 & 2.9 & 8050 & 3.1 & \\
HD 86986 & 0.022 & {$[-1.75 \mathrm{a}]$} & 2.0 & 8100 & 2.8 & 8050 & 3.3 & \\
HD 2857 & 0.022 & {$[-1.75 \mathrm{a}]$} & 4.0 & 7600 & 2.8 & 7550 & 3.0 & \\
HD 161817 & 0.000 & {$[-1.50 \mathrm{a}]$} & 4.0 & 7600 & 2.7 & 7600 & 3.1 & \\
HD 202759 & 0.072 & {$[-2.00 \mathrm{a}]$} & 2.0 & 7500 & 2.8 & 7400 & 3.0 & \\
\hline
\end{tabular}

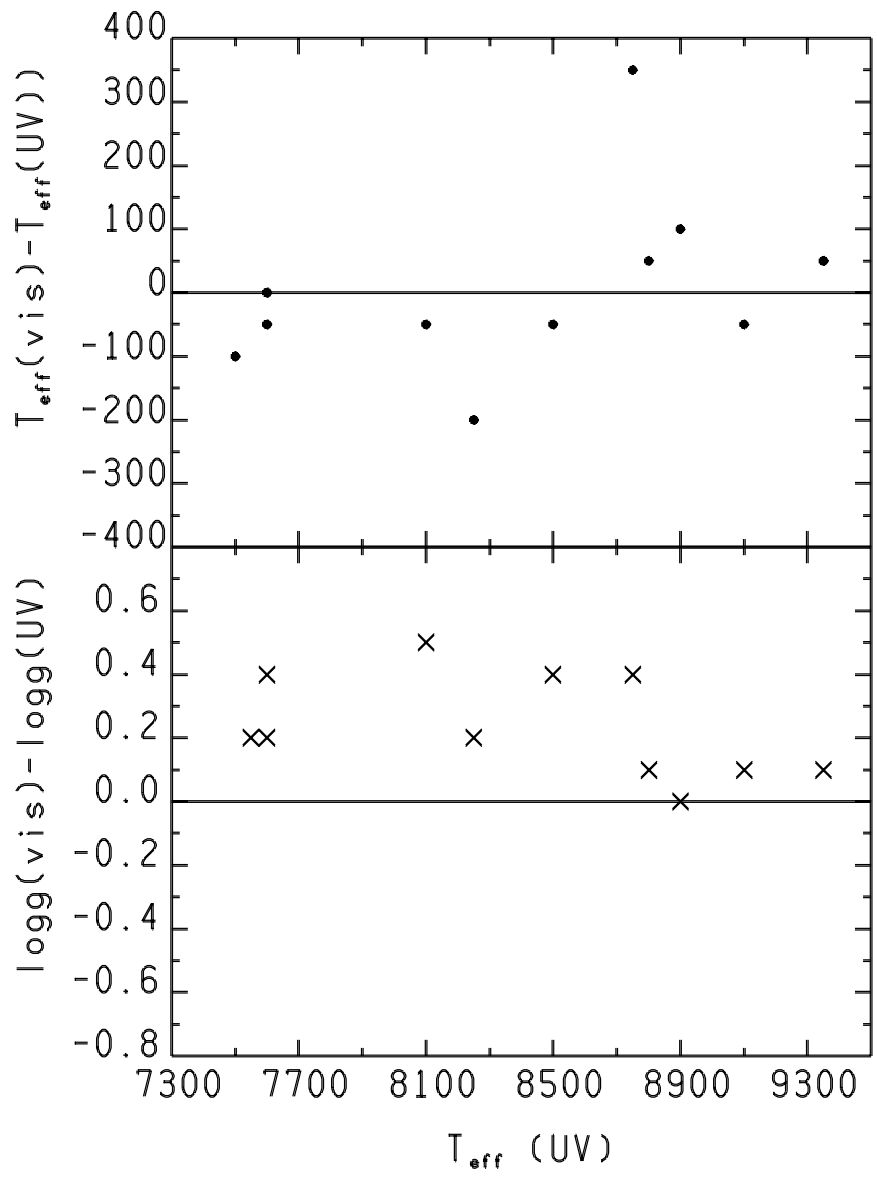

Fig. 6. Upper panel: differences between $T_{\text {eff }}$ derived from visual and ultraviolet energy distributions, as a function of $T_{\text {eff }}$ for stars with available spectrophotometry. Lower panel: same as upper panel, for $\log g$.

For HD 86986, $E(B-V)$ between $0.03 \mathrm{mag}$ and $0.05 \mathrm{mag}$ gives $T_{\text {eff }}$ from the visible flux in agreement within $50 \mathrm{~K}$ with $T_{\text {eff }}$ from the ultraviolet flux, but also for this star, there is always a difference on the order of 0.5 dex between the gravities, whichever is $E(B-V)$.

In conclusion, for the two stars examined, a reddening change may improve the agreement between $T_{\text {eff }}$ derived
Table 5. $E(B-V)$ taken from Huenemoerder et al. (1984) (HBC), Hayes \& Philip (1988) (HP), Gray et al. (1996) (GCP), Gratton (1998) (G), and Altman \& de Boer (2000) (AB) ${ }^{1}$.

\begin{tabular}{lccccc}
\hline Star & HBC & HP & GCP & G & AB \\
\hline BD +00 145 & 0.05 & & & & \\
BD +42 2309 & & 0.000 & 0.001 & & 0.000 \\
HD 2857 & 0.02 & 0.019 & 0.044 & & 0.050 \\
HD 14829 & & 0.012 & 0.015 & & 0.020 \\
HD 31943 & & & & 0.015 & \\
HD 60778 & 0.02 & 0.014 & 0.067 & & 0.020 \\
HD 74721 & 0.02 & 0.014 & 0.000 & 0.000 & 0.000 \\
HD 78913 & & & & & 0.030 \\
HD 86986 & 0.03 & 0.027 & 0.034 & 0.035 & 0.035 \\
HD 93329 & & & & 0.156 & \\
HD 106304 & & & & & 0.040 \\
HD 109995 & 0.00 & 0.000 & 0.001 & 0.001 & 0.001 \\
HD 117880 & 0.02 & 0.014 & 0.067 & & 0.015 \\
HD 128801 & & & & 0.037 & \\
HD 130095 & 0.10 & $(0.055)$ & 0.063 & 0.064 & 0.064 \\
HD 139961 & 0.10 & & & 0.107 & 0.107 \\
HD 161817 & 0.02 & 0.014 & 0.020 & 0.020 & 0.020 \\
HD 167105 & & & & 0.057 & \\
HD 202759 & & 0.068 & & & \\
HD 213468 & 0.02 & & & & \\
\hline
\end{tabular}

${ }^{1}$ Note that Gratton (1998) (G) adopted the reddening from Gray et al. (1996) (GCP) for the stars in common, and Altman \& de Boer (2000) (AB) adopted the reddening from Gratton (1998) for the stars in common.

from the visible and ultraviolet fluxes, but the discrepancy between the gravities does not decrease.

\subsection{The microturbulent velocity, the metallicity, and the mixing-length parameter}

We investigated the effect of different microturbulent velocities $\xi$, different metallicities $[\mathrm{M} / \mathrm{H}]$, and different mixing-length parameters for the convection $L / H_{\mathrm{p}}$ on the parameters of HD 2857 and HD 86986. Table 7 shows 


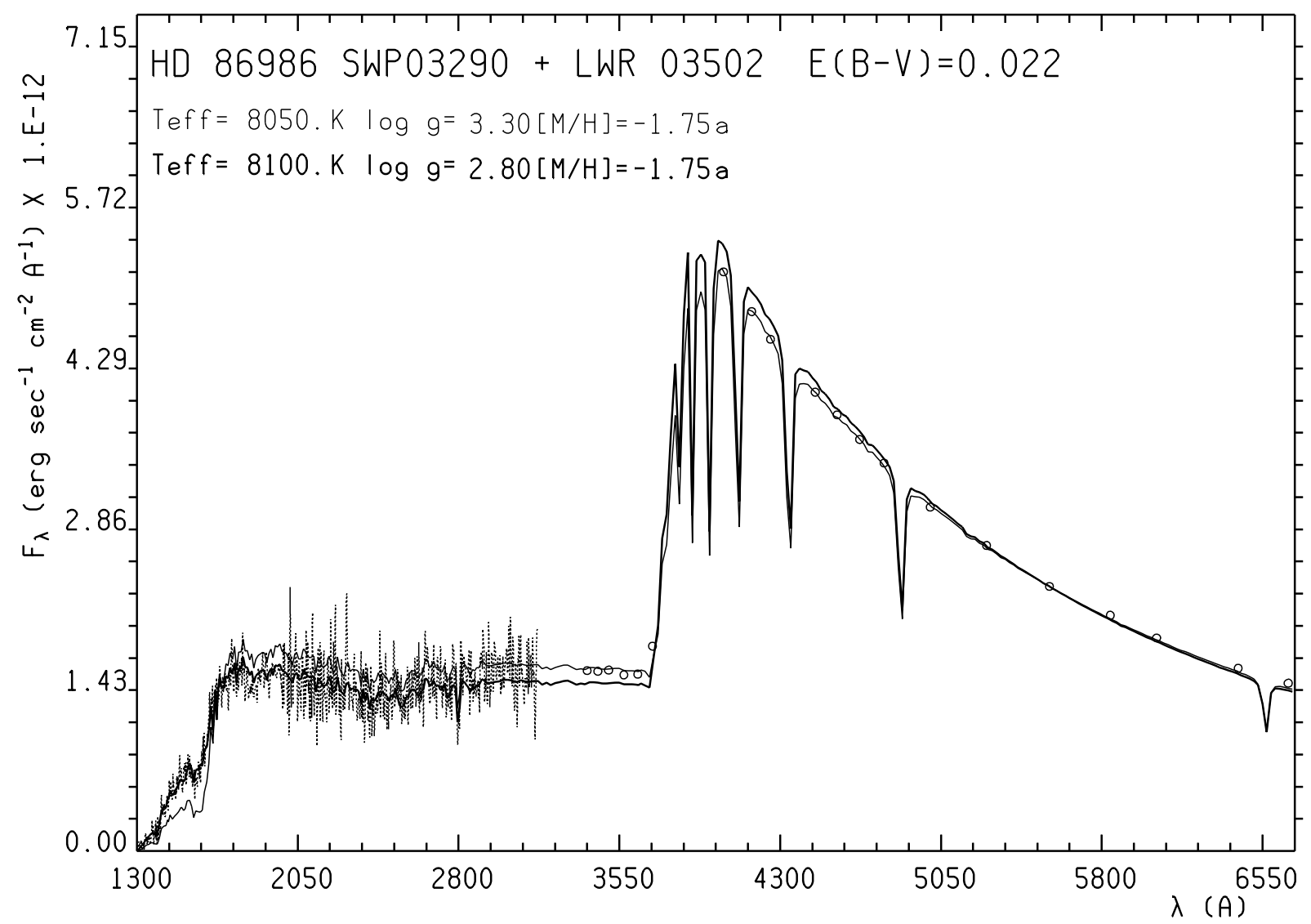

Fig. 7. The ultraviolet energy distribution from IUE (dashed line) and the visual energy distribution from Philip \& Hayes (1983) (open circles) of HD 86986 are compared with the computed flux which fits the ultraviolet observations $\left(T_{\text {eff }}=8100 \mathrm{~K}\right.$, $\log g=2.8,[\mathrm{M} / \mathrm{H}]=-1.75 \mathrm{a})$ (thick line) and with the computed flux which fits the visual observations $\left(T_{\text {eff }}=8150 \mathrm{~K}, \log g=3.3\right.$, $[\mathrm{M} / \mathrm{H}]=-1.75 \mathrm{a})$ (thin line).

that changes in microturbulent velocity, metallicity, and mixing-length parameters modify $T_{\text {eff }}$ and $\log g$ within the uncertainty limits of the fitting procedure, namely no more than $50 \mathrm{~K}$ and $0.1 \mathrm{dex}$, respectively. Therefore, the discrepancy in $\log g$ derived from the ultraviolet and visible fluxes does not change by changing $\xi,[\mathrm{M} / \mathrm{H}]$, or $L / H_{\mathrm{p}}$.

\section{Comparison of $T_{\text {eff }}$ and $\log g$ with other determinations and with the evolutionary models}

In order to have an idea whether the gravities we derived from the ultraviolet energy distribution are more reliable than those we derived from the visible region in the case of stars cooler than about $8700 \mathrm{~K}$, we have compared our determinations with those from other sources and with the evolutionary models plotted in the $T_{\text {eff }}, \log g$ plane.

\subsection{Comparison with other sources}

Table 8 compares the parameters derived by us from the ultraviolet energy distribution (Cols. 4, 5) and from the visible energy distribution (Cols. 6,7 ) with the parameters from Wilhelm et al. (1999) (WBG) (Cols. 9, 10) and with those from Gray et al. (1996) (GCP) (Cols. 13, 14). The parameters from WBG are based on both $U B V$ photometry and a spectroscopic index $D_{0.2}$, related with the width of the $\mathrm{H}_{\gamma}$ and $\mathrm{H}_{\delta}$ Balmer profiles, which were measured by WBG on medium-resolution spectra. The parameters from GCP are based on both Strömgren photometry and the index $D_{0.2}$, which was measured by GCP on $2.8 \AA$ resolution spectra.

The comparison of the parameters derived by us with those from WBG is shown in Fig. 8, where the stars are plotted in the $T_{\text {eff }}, \log g$ plane. The upper panel in Fig. 8 indicates that the parameters from WBG (full points) and the parameters derived by us from the ultraviolet energy distribution (open triangles) agree quite well, with only one exception, HD 180903. The lower panel of Fig. 8 shows that the gravities derived by us from the visible energy distributions (open triangles) are larger than the gravities derived by WBG (full points).

Figure 9 compares the parameters derived by us with those derived by GCP. In this case, the upper plot of Fig. 9 shows poor agreement between the parameters derived by us from the IUE spectra (open triangles) and those from GCP (full points), in the sense that the gravities derived from the ultraviolet energy distributions are systematically lower. Vice versa, the lower panel of Fig. 9 shows 
Table 6. The effect of different choices of $E(B-V)$ on model parameters derived from UV (1200-1978 $\AA$ ) and from the visible $(3400-7000 \AA)$ for HD $2857([M / H]=-1.75 \mathrm{a}$, $\left.\xi=4 \mathrm{~km} \mathrm{~s}^{-1}\right)$ and HD $86986\left([\mathrm{M} / \mathrm{H}]=-1.75 \mathrm{a}, \xi=2 \mathrm{~km} \mathrm{~s}^{-1}\right)$.

\begin{tabular}{ccccc}
\hline$E(B-V)$ & $T_{\text {eff }}$ & $\log g$ & $T_{\text {eff }}$ & $\log g$ \\
\hline \multicolumn{4}{c}{ HD 2857} \\
UV \\
\hline 0.00 & 7600 & 2.3 & 7350 & 2.8 \\
0.01 & 7600 & 2.5 & 7450 & 2.9 \\
0.02 & 7650 & 2.5 & 7550 & 3.0 \\
0.03 & 7700 & 2.6 & 7550 & 3.1 \\
0.04 & 7700 & 2.7 & 7650 & 3.2 \\
0.05 & 7750 & 2.8 & 7700 & 3.2 \\
0.06 & 7750 & 2.9 & 7800 & 3.3 \\
0.07 & 7800 & 3.0 & 7900 & 3.4 \\
0.08 & 7800 & 3.1 & 7950 & 3.5 \\
\hline
\end{tabular}

\begin{tabular}{lllll}
\hline \multicolumn{4}{c}{ HD 86986} \\
UV & \multicolumn{2}{c}{ visible } \\
\hline 0.00 & 8050 & 2.5 & 7800 & 3.1 \\
0.01 & 8050 & 2.6 & 7900 & 3.1 \\
0.02 & 8100 & 2.7 & 8000 & 3.2 \\
0.03 & 8100 & 2.8 & 8100 & 3.3 \\
0.04 & 8150 & 2.9 & 8200 & 3.4 \\
0.05 & 8200 & 3.1 & 8250 & 3.4 \\
0.06 & 8250 & 3.1 & 8400 & 3.5 \\
0.07 & 8250 & 3.2 & 8550 & 3.4 \\
0.08 & 8300 & 3.4 & 8700 & 3.4 \\
\hline
\end{tabular}

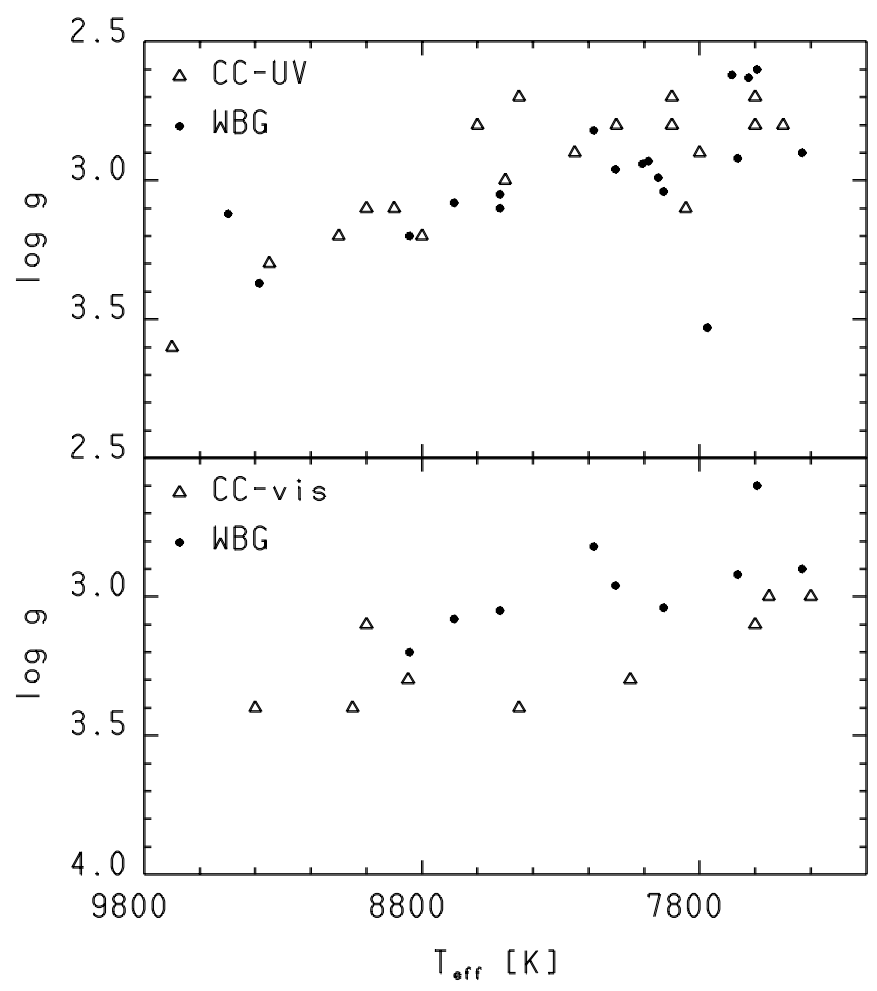

Fig. 8. Comparison of the parameters from Wilhelm et al. (1999) (WBG) (full points) with the parameters from this paper (CC) (open triangles) from the ultraviolet energy distribution (upper panel) and from the visible energy distribution (lower panel).

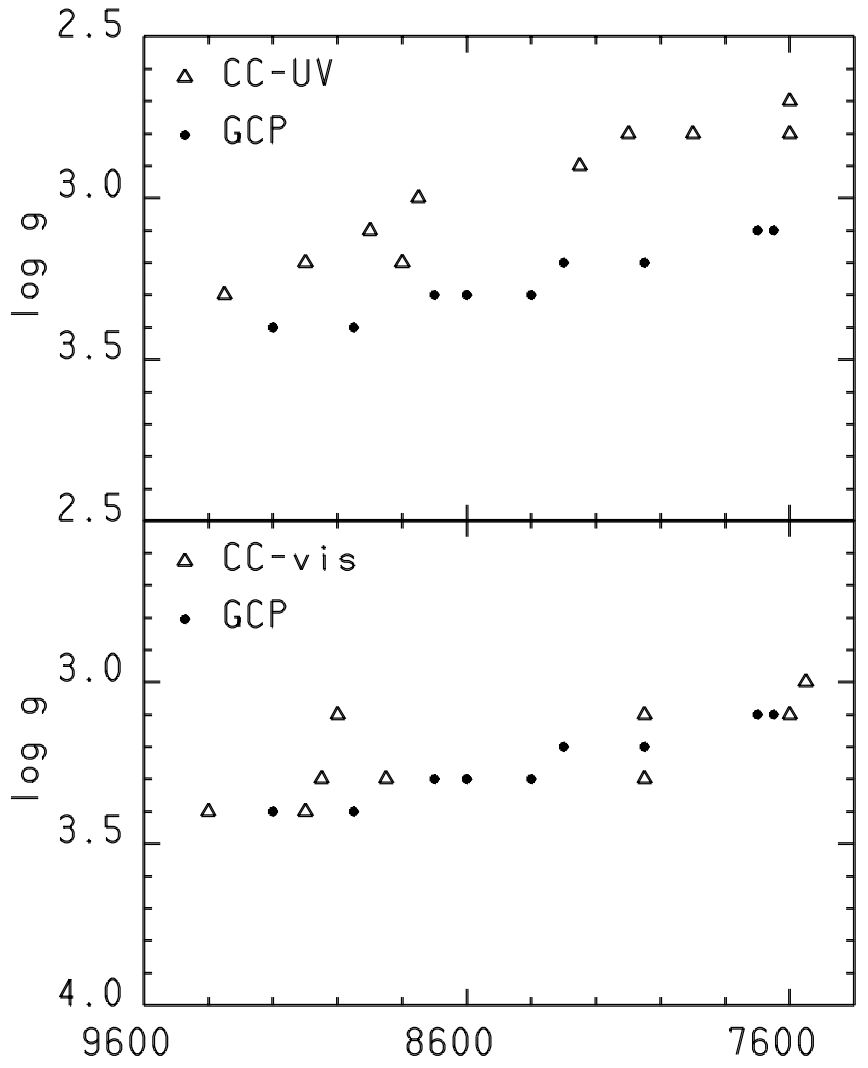

Fig. 9. Comparison of the parameters from Gray et al. (1996) (GCP) (full points) with the parameters from this paper (CC) (open triangles) from the ultraviolet energy distribution (upper plot) and from the visible energy distribution (lower panel).

that the gravities derived by us from the visible region well agree with those from GCP.

In conclusion, the comparison of the parameters derived by us with those from WBG and GCP has not helped solving the question whether the gravities from the ultraviolet energy distributions are more reliable than those from the visible energy distributions.

\subsection{The comparison with the evolutionary models}

The parameters derived for our target stars from the IUE spectra and the new-ODF models (Cols. 5 and 6 of Table 3) are compared in Fig. 10 with ZAHB models at metallicity $Z=0.002$ and primordial helium content $Y=0.23,0.33$ and 0.43 . These models are discussed in Sect. 3.1 of Sweigart \& Catelan (1998), and were kindly made available to us by Catelan (2001, private communication). The stars BD +32 2188 and BD +00 145 are not shown in Fig. 10 as they are not BHB stars.

This comparison shows that, with the exception of the few stars hotter than about $9500 \mathrm{~K}$, the gravities that we derive would be appropriate for models with a (unrealistically) high content of primordial helium. Other mechanisms of helium enhancement, such as those proposed by Sweigart (1999) and related to non-canonical mixing, are not relevant here because no significant mixing is expected 
Table 7. The effect of different choices of $\xi$, [M/H], and $L / H_{\mathrm{p}}$ on model parameters derived from UV (1200-1978 $\left.\AA\right)$ and from the visible $(3400-700 \AA)$ energy distribution for HD 2857 when $E(B-V)=0.022$, and for HD 86986 when $E(B-V)=0.022$.

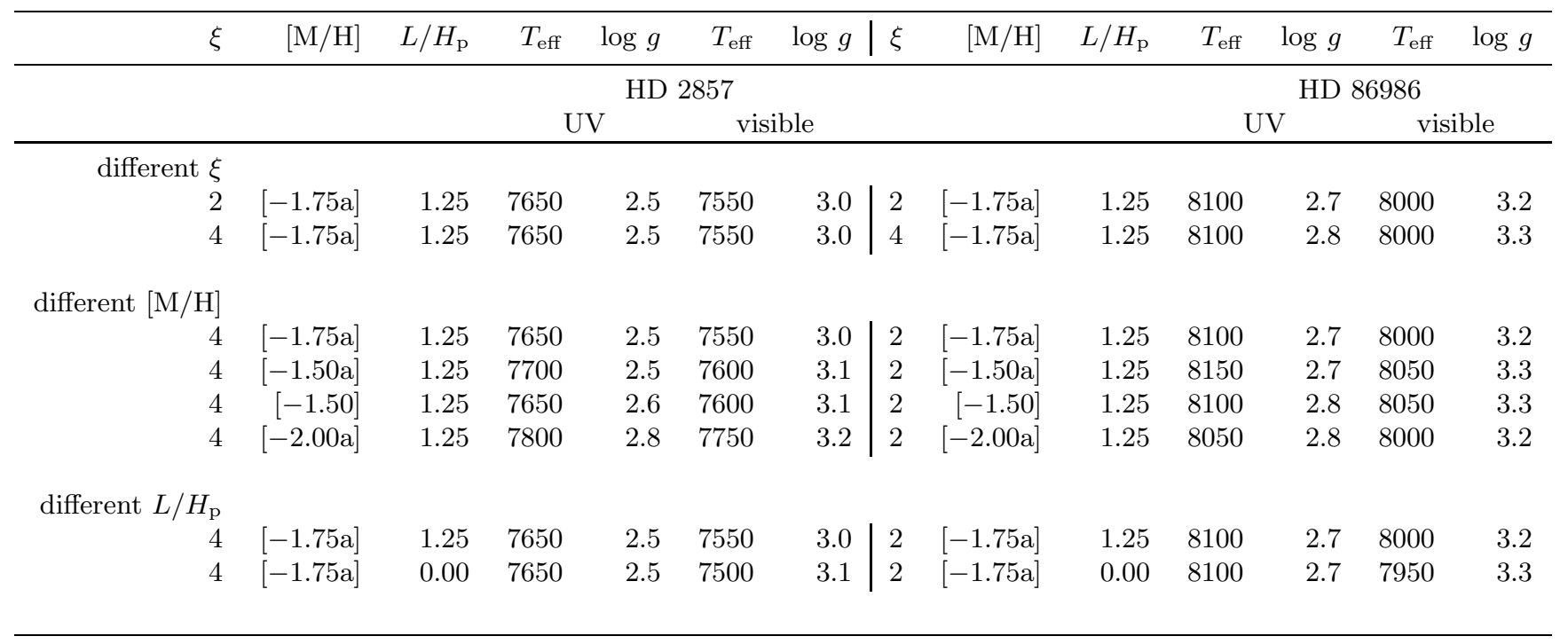

Table 8. Comparison between parameters from this paper and those from Wilhelm et al. (1999) (WBG) and from Gray et al. (1996) (GCP).

\begin{tabular}{|c|c|c|c|c|c|c|c|c|c|c|c|c|c|}
\hline \multirow[t]{2}{*}{ Star } & \multirow{2}{*}{$\begin{array}{r}E(B-V) \\
(2)\end{array}$} & \multirow{2}{*}{$\begin{array}{r}{[\mathrm{M} / \mathrm{H}]} \\
(3)\end{array}$} & \multicolumn{2}{|c|}{$\begin{array}{c}T_{\text {eff }} \log g \\
\text { UV }\end{array}$} & \multicolumn{2}{|c|}{$\begin{array}{c}T_{\text {eff }} \log g \\
\text { vis. }\end{array}$} & \multirow{2}{*}{$\begin{array}{r}{[\mathrm{M} / \mathrm{H}]} \\
(8)\end{array}$} & \multirow{2}{*}{$\begin{array}{r}T_{\text {eff }} \\
\text { WBG } \\
(9)\end{array}$} & \multirow{2}{*}{$\begin{array}{r}\log g \\
(10)\end{array}$} & \multirow{2}{*}{$\begin{array}{r}E(B-V) \\
(11)\end{array}$} & \multirow{2}{*}{$\begin{array}{r}\mathrm{M} / \mathrm{H}] \\
\mathrm{GCP} \\
(12)\end{array}$} & \multirow{2}{*}{$\begin{array}{l}T_{\text {eff }} \\
(13)\end{array}$} & \multirow{2}{*}{$\begin{array}{l}\log g \\
(14)\end{array}$} \\
\hline & & & $(4)$ & $(5)$ & (6) & (7) & & & & & & & \\
\hline HD 2857 & 0.022 & {$[-1.75 \mathrm{a}]$} & 7600 & 2.8 & 7700 & 3.2 & {$[-1.62]$} & 7663 & 2.92 & 0.044 & {$[-1.5]$} & 7700 & 3.1 \\
\hline HD 4850 & 0.009 & {$[-1.25 \mathrm{a}]$} & 8450 & 2.7 & & & {$[-1.84]$} & 8006 & 2.94 & & & & \\
\hline HD 13780 & 0.000 & {$[-1.50 \mathrm{a}]$} & 7900 & 2.7 & & & {$[-1.81]$} & 7684 & 2.62 & & & & \\
\hline HD 14829 & 0.018 & {$[-2.00 \mathrm{a}]$} & 8900 & 3.1 & 9000 & 3.1 & {$[-2.42]$} & 8519 & 3.05 & 0.015 & {$[-2.0]$} & 8700 & 3.3 \\
\hline HD 31943 & 0.006 & {$[-1.00 \mathrm{a}]$} & 7850 & 3.1 & & & {$[-1.65]$} & 7624 & 2.63 & & & & \\
\hline HD 60778 & 0.028 & {$[-1.50 \mathrm{a}]$} & 8250 & 2.9 & 8050 & 3.1 & & & & 0.067 & {$[-1.0]$} & 8600 & 3.3 \\
\hline HD 74721 & 0.012 & {$[-1.50 \mathrm{a}]$} & 8800 & 3.2 & 8850 & 3.3 & {$[-2.22]$} & 8181 & 2.82 & 0.000 & {$[-1.5]$} & 8600 & 3.3 \\
\hline HD 86986 & 0.022 & {$[-1.75 \mathrm{a}]$} & 8100 & 2.8 & 8050 & 3.3 & {$[-2.01]$} & 7930 & 3.04 & 0.035 & {$[-1.5]$} & 8050 & 3.2 \\
\hline HD 87047 & 0.006 & {$[-2.50 \mathrm{a}]$} & 7900 & 2.8 & & & {$[-2.11]$} & 7985 & 2.93 & 0.001 & {$[-1.5]$} & 8300 & 3.2 \\
\hline HD 87112 & 0.003 & {$[-1.50 \mathrm{a}]$} & 9700 & 3.6 & & & {$[-1.88]$} & 9386 & 3.37 & & & & \\
\hline HD 93329 & 0.014 & {$[-1.50 \mathrm{a}]$} & 8250 & 2.9 & & & {$[-1.85]$} & 7949 & 2.99 & & & & \\
\hline HD 109995 & 0.010 & {$[-1.75 \mathrm{a}]$} & 8500 & 3.0 & 8450 & 3.4 & {$[-2.11]$} & 8103 & 2.96 & & & & \\
\hline HD 117880 & 0.077 & {$[-1.50 \mathrm{a}]$} & 9350 & 3.3 & 9400 & 2.8 & {$[-2.53]$} & 8684 & 3.08 & 0.067 & {$[-1.5]$} & 9200 & 3.4 \\
\hline HD 130095 & 0.072 & {$[-2.00 \mathrm{a}]$} & 9100 & 3.2 & 9050 & 3.4 & {$[-2.34]$} & 8845 & 3.20 & 0.064 & {$[-1.5]$} & 8950 & 3.4 \\
\hline HD 139961 & 0.051 & {$[-1.75 \mathrm{a}]$} & 8600 & 2.8 & & & {$[-1.68]$} & 8519 & 3.10 & & & & \\
\hline HD 161817 & 0.000 & {$[-1.50 \mathrm{a}]$} & 7600 & 2.7 & 7600 & 3.1 & {$[-1.67]$} & 7593 & 2.60 & 0.020 & {$[-1.2]$} & 7650 & 3.1 \\
\hline HD 167105 & 0.024 & {$[-1.50 \mathrm{a}]$} & 9000 & 3.1 & & & {$[-1.95]$} & 9498 & 3.12 & & & & \\
\hline HD 180903 & 0.098 & {$[-1.50 \mathrm{a}]$} & 7800 & 2.9 & & & {$[-1.44]$} & 7772 & 3.53 & & & & \\
\hline HD 202759 & 0.072 & {$[-2.00 \mathrm{a}]$} & 7500 & 2.8 & 7400 & 3.0 & {$[-2.37]$} & 7431 & 2.90 & & & & \\
\hline $\mathrm{BD}+422309$ & 0.013 & {$[-1.75 \mathrm{a}]$} & 8750 & 3.0 & 9100 & $(2.4)$ & & & & 0.001 & {$[-1.5]$} & 8400 & 3.3 \\
\hline
\end{tabular}

to occur in field stars, and in any case i) it would affect the hotter stars rather than the cooler ones; and ii) it would produce abundance anomalies that are not observed in our sample.

This result leads us to conclude that determinations of gravity, as difficult as they may be in any wavelength range, are particularly uncertain and unreliable in the UV range especially for stars cooler than about $8700 \mathrm{~K}$.

\section{Conclusions}

We derived the parameters $T_{\text {eff }}$ and $\log g$ from the whole IUE energy distribution for 29 out of the 31 metal-poor A-type stars studied by Kinman et al. (2000) (KCC). We used both the same models adopted by KCC and new models computed with ODFs which take into account the $\mathrm{H}-\mathrm{H}$ and $\mathrm{H}-\mathrm{H}^{+}$semi-molecular absorptions at $1400 \AA$ 


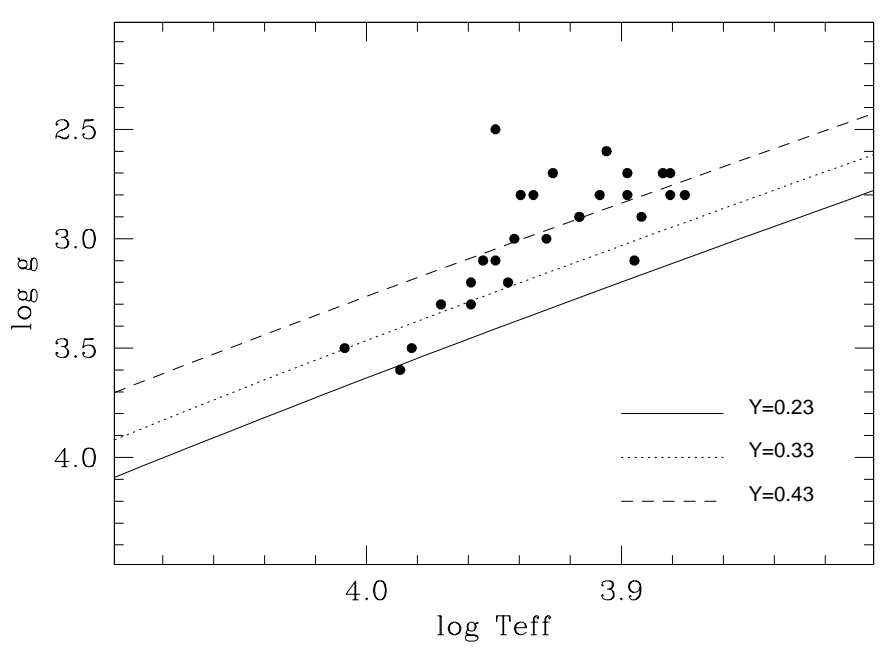

Fig. 10. The parameters for the BHB stars derived from the UV energy distributions and new-ODF models (dots) are compared with the ZAHB evolutionary tracks for $Z=0.002$ and primordial helium abundances $Y=0.23,0.33$ and 0.43 (Catelan 2001, private communication).

and $1600 \AA$, whose intensity strongly depends on $T_{\text {eff }}$ and $\log g$.

For most stars, the new-ODF models and the old-ODF models lead pratically to the same values of $T_{\text {eff }}$ and $\log g$, when the fit is performed in the UV range $1200-3300 \AA$; the difference is within the uncertainty of the fit of the observations to the models, namely $50 \mathrm{~K}$ in $T_{\text {eff }}$ and 0.1 dex in $\log g$. Larger differences in $\log g$ on the order of 0.2 dex or more have been found only for few stars. However, a detailed inspection of the figures in Appendix A points out the better fit provided by the new-ODF models, especially for the coolest stars in the sample, i.e. those from HD 8376 (Fig. A.11) to HD 202759 (Fig. A.15). For all the stars, the computed energy distribution shortward of $1600 \AA$ is no longer brighter than the models, so that the shortcoming pointed out by Huenemorder et al. (1984) has been completely overcome by the new models.

HD 130201 is the only star in the sample with both parameters from the whole ultraviolet energy distribution in clear disagreement with those from KCC $\left(\Delta T_{\text {eff }}=250 \mathrm{~K}\right.$, $\Delta \log g=1.0)$. For all the other stars hotter than about $8700 \mathrm{~K}$ the parameters from KCC agree with those from the whole IUE spectra within $150 \mathrm{~K}$ in $T_{\text {eff }}$ and 0.2 dex in $\log g$. We did not observe any trend in $\Delta T_{\text {eff }}$ or $\Delta \log g$ as a function of $T_{\text {eff }}$ for these stars. Also for stars cooler than about $8700 \mathrm{~K}$ the differences in $T_{\text {eff }}$ are not larger than $150 \mathrm{~K}$ (except for HD 60788 and HD 78913, with $\Delta T_{\text {eff }}=200 \mathrm{~K}$ ), whereas the gravities derived from fitting the UV energy distributions to the models are systematically smaller than the gravities obtained from photometric and spectrophotometric data in the visual range. The average difference is about $0.3 \mathrm{dex}$. This discrepancy in $\log g$ is insensitive to reddening, microturbulent velocity, metallicity, and mixing-length parameter for the treatment of the convection.
The comparison of the parameters derived in this paper with those derived from two independent data sets and analyses in the visual range (Wilhelm et al. 1999; Gray et al. 1996) does not allow to decide whether the problem resides in the UV or in the visual range, since our parameters from UV agree with Wilhelm et al.'s (1999) and do not agree with Gray et al.'s (1996).

On the other hand, a comparison with ZAHB models (based on Sweigart \& Catelan 1998) at metallicity $Z=0.002$ and various values of primordial helium content indicates that the gravities derived from UV data are too low for stars cooler than about $9000 \mathrm{~K}$.

We conclude that fitting the most recent model atmospheres to IUE ultraviolet energy distributions yields reliable values of $T_{\text {eff }}$ and $\log g$ for HB A-type stars hotter than about $8700 \mathrm{~K}$, whereas for cooler stars only $T_{\text {eff }}$ is acceptable.

Further investigations are needed in order to understand why the present model atmospheres yield such discrepant results on gravities in the UV and visual ranges at $T_{\text {eff }}$ lower than about $8700 \mathrm{~K}$. A possibility is that the classical LTE models are inadequate to represent the atmospheres of the cooler HB A-type stars. For instance, both NLTE and convection can play an important role in the modelisation of the metal-poor, low gravity A-type stars investigated in this paper. Also, missing lines in the ultraviolet, as well as different elemental abundances from those adopted by us (with the exception of $\mathrm{Mg}, \mathrm{Ti}$, and $\mathrm{Fe}$ ) could be the cause for the inconsistency in the gravities. New spectrophotometric observations in the visible and high-resolution spectroscopic observations, mostly in the UV, would be very useful in order to better define possible deficiencies of the models for the HB A-type stars.

Acknowledgements. We wish to thank T. Kinman and M. Catelan for useful comments on the manuscript, and M. Catelan for providing us with the ZAHB unpublished models discussed by Sweigart \& Catelan (1998).

\section{Appendix A: Comparison of the IUE fluxes of the Pop II A-type stars with the computed fluxes}

The figures in this Appendix are only available in electronic form at http://www. edpsciences.org

Figures A.1-A.15 compare the IUE low-resolution spectra with the computed spectra for the sample of stars listed in Table 3. Dashed lines indicate the observed fluxes. Two vertical lines in the plots give the position of the Lyman- $\alpha$ H-H ${ }^{+}$and H-H quasi-molecular absorptions at $1400 \AA$ and $1600 \AA$, respectively. For each star there are two plots, plot "OLD" and plot "NEW". In the plot "OLD" the thin line is the old-ODF flux computed with model parameters $T_{\text {eff }}$ and $\log g$ from KCC (Cols. 9 and 10 in Table 3), while the thick line is the flux computed with parameters derived by fitting the IUE spectra to the grid of fluxes computed with the old-ODFs (Cols. 7 and 8 in Table 3). In the plot "NEW" the thick 
line is the flux computed with parameters derived by fitting the observed fluxes to the grid of fluxes computed with the new-ODFs (Cols. 5 and 6 in Table 3).

Observed and computed fluxes are in units erg s ${ }^{-1} \mathrm{~cm}^{-2} \AA^{-1}$ and are normalized at $5556 \AA$.

Thick lines in the plots "OLD" of Figs. A.1-A.15 show that the fluxes computed from old-ODF models reproduce the IUE spectrum in the whole $1200-3000 \AA$ interval, except around $1400 \AA$ and $1600 \AA$ when the quasi-molecular absorptions $\mathrm{H}-\mathrm{H}^{+}$and $\mathrm{H}-\mathrm{H}$ are observed. Thick lines in the plots "NEW" of Figs. A.1-A.15 show that the models computed with the new-ODFs improve the agreement at $1400 \AA$ and $1600 \AA$ between the observed and computed fluxes. They usually well reproduce the whole observed energy distribution from $1200 \AA$ to $3000 \AA$. Only for a few stars, like BD +00 145 (Fig. A.2) and HD 2857 (Fig. A.14) the models with parameters derived from the whole IUE spectra do not reproduce the observed quasimolecular absorptions. For these two stars, we show in the plot "NEW" both the computed flux which fits the whole IUE spectrum and the computed flux which fits only the short-wavelength spectrum.

The Figs. A.1-A.15 indicate that the $\mathrm{H}-\mathrm{H}^{+}$absorption at $1400 \AA$ is very weak around $10000 \mathrm{~K}$, increases with decreasing temperature up to about $8500 \mathrm{~K}$ and then decreases starting from about $T_{\text {eff }}<8500 \mathrm{~K}$. Stars with a remarkable depression at $1400 \AA$ are HD 167105 (Fig. A.5) and HD 14829 (Fig. A.5).

The H-H absorption at $1600 \AA$ can be observed starting from about $T_{\text {eff }}=8700 \mathrm{~K}$. It increases with decreasing $T_{\text {eff }}$ and with decreasing metallicity. Stars with a remarkable depression at $1600 \AA$ are HD 86986 (Fig. A.10), HD 8376 (Fig. A.11), HD 13780 (Fig. A.11), HD 87047 (Fig. A.12), and HD 202759 (Fig. A.15).

\section{References}

Allard, N. F., Drira, I., Gerbaldi, M., Kielkopf, J., \& Spielfiedel, A. 1998, A\&A, 335, 1124
Altman, M., \& de Boer, K. 2000, A\&A, 353, 135

Anders, A., \& Grevesse, N. 1989, Geochim. Cosmochim. Acta, 53,197

Cacciari, C., Malagnini, M. L., Morossi, C., \& Rossi, L. 1987, A\&A, 183, 314

Castelli, F. 1999, A\&A, 346, 564

Castelli, F., \& Kurucz, R. L. 2001, A\&A, 372, 260

Crawford, D. L., \& Mandwewala, N. 1976, PASP, 88,917

Gratton, R. G. 1998, MNRAS, 296, 739

Gray, D. F. 1976, The Observation and the Analysis of Stellar Photospheres (Cambridge Univ. Press, Cambridge)

Gray, R. O., Corbally, C. J., \& Philip, A. G. D. 1996, AJ, 112, 2291

Grevesse, N., Noels, A., \& Sauval, A. J. 1996, Cosmic Abundances, ed. S. S. Holt, \& G. Sonnebord, ASP Conf. Ser., 99, 117

Hayes, D. S., \& Philip, A. G. D. 1988, PASP, 100, 801

Holweger, H., Koester, D., \& Allard, N. F. 1994, A\&A, 290, L21

Holweger, H., Kock, M., \& Bard, A. 1995, A\&A, 296, 233

Huenemoerder, D. P., de Boer, K. S., \& Code, A. D. 1984, AJ, 89,851

INES newsletter, March 2000, ESA Publication Division

Jaschek, M., Baschek, B., Jaschek, C., \& Heck, A. 1985, A\&A, 152,439

Kinman, T., Castelli, F., Cacciari, C., et al. 2000, A\&A, 364, 102

Kurucz, R. L. 1979a, ApJS, 40, 1

Kurucz, R. L. 1979b, Model Atmospheres for G, F, A, B, and O Stars (magnetic tape version with unpublished models)

Kurucz, R. L. 1990, Stellar Atmospheres: Beyond Classical Models, NATO Asi Ser., ed. L. Crivellari et al., 441

Kurucz, R. L. 1993, ATLAS9 Stellar Atmosphere Programs and $2 \mathrm{~km} / \mathrm{s}$ grid, CD-ROM, No. 13

Lane, M. C., \& Lester, J. B. 1984, ApJ, 281, 723

Mathis, J. S. 1990, ARA\&A, 28, 37

Philip, A. G. D., \& Hayes, D. S. 1983, ApJS, 53, 751

Sweigart, A. V. 1999, in The 3rd Conf. on Faint Blue Stars, ed. A. G. D. Philip, J. Liebert, \& R. A. Saffer (Schenectady: L. Davis Press), 3

Sweigart, A. V., \& Catelan, M. 1998, ApJ, 501, L63

Wilhelm, R., Beers, T. C., \& Gray, R. O. 1999, ApJ, 117, 2308 